\title{
Solar Magnetic Carpet III: Coronal Modelling of Synthetic Magnetograms
}

\author{
K.A. Meyer $^{1}$ - D.H. Mackay ${ }^{1}$. \\ A.A. van Ballegooijen ${ }^{2}$. C.E. Parnell ${ }^{1}$ \\ Received 21 September 2012 / accepted 5 March 2013 \\ (C) Springer $\bullet \bullet \bullet \bullet$
}

\begin{abstract}
This paper is the third in a series of papers working towards the construction of a realistic, evolving, non-linear force-free coronal field model for the solar magnetic carpet. Here, we present preliminary results of 3D time-dependent simulations of the small-scale coronal field of the magnetic carpet. Four simulations are considered, each with the same evolving photospheric boundary condition: a 48 hr time series of synthetic magnetograms produced from the model of Meyer et al. (2011). Three simulations include a uniform, overlying coronal magnetic field of differing strength, the fourth simulation includes no overlying field. The buildup, storage and dissipation of magnetic energy within the simulations is studied. In particular, we study their dependence upon the evolution of the photospheric magnetic field and the strength of the overlying coronal field. We also consider where energy is stored and dissipated within the coronal field. The free magnetic energy built up is found to be more than sufficient to power small-scale, transient phenomena such as nanoflares and X-ray bright points, with the bulk of the free energy found to be stored low down, between $0.5-0.8 \mathrm{Mm}$. The energy dissipated is presently found to be too small to account for the heating of the entire quiet Sun corona. However, the form and location of energy dissipation regions are in qualitative agreement with what is observed on small scales on the Sun. Future MHD modelling using the same synthetic magnetograms may lead to a higher energy release.
\end{abstract}

Keywords: Sun: magnetic fields - Sun: magnetic carpet

\footnotetext{
1 School of Mathematics and Statistics, University of St Andrews, North Haugh, St Andrews, Fife, KY16 9SS, Scotland, U.K. 


\section{Introduction}

The small-scale photospheric magnetic field of the quiet Sun, the magnetic carpet, is complex and constantly evolving. This evolution is due to underlying photospheric flows produced by convection on many scales (e.g. granulation and supergranulation, Leighton, Noyes, and Simon (1962), Wang and Zirin (1989), Schrijver, Hagenaar, and Title (1997), Rieutord and Rincon (2010)) and the flux evolution processes of emergence, cancellation, coalescence and fragmentation. Small-scale magnetic features classified as ephemeral regions (Harvey and Martin, 1973, Harvey, 1993, Hagenaar, DeRosa, and Schrijver, 2008, Schrijver, 2010), internetwork features (Livingston and Harvey, 1975 |Wang et al., 1996; de Wijn et al., 2008; Zhou et al., 2010) and network features (Simon and Leighton, 1964 Zirin, 1985, Martin, 1984, Martin, 1988) continually interact with one another, resulting in a photospheric recycle time of just $1-2 \mathrm{hr}$ (hours) (Hagenaar, DeRosa, and Schrijver, 2008). This is the time taken for all flux within the quiet Sun photosphere to be replaced. Since magnetic fields from the magnetic carpet extend up into the solar chromosphere and lower corona, it is expected that the quiet Sun corona is also highly dynamic. Complex interactions of magnetic features on the photosphere may result in significant heating of the corona, for example through braiding and reconnection of magnetic field lines (e.g. Galsgaard and Nordlund (1996), Parnell and Galsgaard (2004), Haynes et al. (2007) Rappazzo et al. (2008), Berger and Asgari-Targhi (2009), Wilmot-Smith, Hornig, and Priest (2009), Pontin et al. (2011)). Therefore, it is of interest to simulate the small-scale coronal field resulting from the evolution of the solar magnetic carpet.

Previous magnetic carpet coronal field models using extrapolation methods have studied, for example, flux topology and connectivity (Schrijver and Title, 2002 Close et al., 2003); the number density and locations of coronal null points (Schrijver and Title, 2002, Régnier, Parnell, and Haynes, 2008; Longcope and Parnell, 2009); coronal remap times (Close et al., 2004: Cranmer and van Ballegooijen, 2010); and whether the solar wind can be driven by reconnection in the magnetic carpet (Cranmer and van Ballegooijen, 2010). However, each of these studies considered only potential field extrapolations of the small-scale coronal field.

Within this paper, we present preliminary results of $3 \mathrm{D}$ simulations of the network-scale coronal field of the magnetic carpet. In contrast to the models described above, which produce independent potential field extrapolations, we model a continuous evolution of a non-linear force-free coronal field. A non-linear force-free field satisfies the conditions $\nabla \cdot \mathbf{B}=0$ and $\nabla \times \mathbf{B}=\alpha \mathbf{B}$, where $\alpha=\alpha(\mathbf{r})$ is a scalar function of position (but constant along a given field line) describing the twist of the field. This approximation is a step up in complexity from a potential field as it allows for the existence of electric currents and free magnetic energy. In Meyer et al. (2011) (hereafter Paper I) we presented a realistic model for the photospheric evolution of the magnetic carpet, that reproduced many observational properties. Synthetic magnetograms produced from this model will provide the photospheric boundary condition to drive the evolution of the full $3 \mathrm{D}$ coronal field. The coronal field is evolved through a series of quasi-static, nonlinear force-free states in response to the evolution of the photospheric magnetic 
field, using a magnetofrictional technique that is described in Meyer, Mackay, and van Ballegooijen (2012) (hereafter Paper II). There are several advantages to using synthetic magnetograms as opposed to actual observed data: (i) The total magnetic flux within the synthetic magnetograms is always known - there is no noise or instrumental limitations. (ii) We have complete control over the photospheric evolution - magnetic features do not drift into or out of the field of view, which is unavoidable in real magnetograms. (iii) The synthetic magnetograms are always in flux balance (total positive flux $=$ total negative flux). This is a requirement for the coronal model due to our set-up. (iv) We know exactly where and when each of the processes of emergence, cancellation, coalescence and fragmentation occur within the synthetic magnetograms, as well as the exact flux involved in each event. Many feature tracking techniques exist to follow the evolution of magnetic features in real magnetogram data, e.g. DeForest et al. (2007). However, these are limited by factors such as spatial resolution and time cadence. In a future study, we will consider in detail how various photospheric events in our synthetic magnetograms affect the evolution of the coronal field.

In this paper we consider four simulations in total, each with the same photospheric boundary condition: a $48 \mathrm{hr}$ set of synthetic magnetograms. Three of the simulations have a uniform, overlying coronal magnetic field (of varying strength) which points in the positive $x$-direction; this simulates the influence of larger scale magnetic features, such as exist on the Sun, especially during solar maximum. The fourth simulation has no overlying field and thus represents a very quiet region of the Sun, such as may be found during solar minimum. We consider the magnetic energy both stored and dissipated within the coronal volume, along with the square of the current density, $j^{2}$. Some of the properties of these quantities that we consider are: how they evolve in time, where they are located spatially with respect to the underlying photospheric magnetic field, and the effect of varying the strength of the overlying coronal magnetic field.

Section 2 describes the lower boundary condition and the set-up of the 3D simulations. Section 3 provides the results, while Section 4 gives a discussion and conclusions.

\section{Lower Boundary Condition and Set-Up}

We choose the most realistic simulation from Paper I to provide the lower boundary condition for 3D modelling. This simulation, which covers an area of $50 \times 50 \mathrm{Mm}^{2}$, is of length $250 \mathrm{hr}$ with a cadence of $1 \mathrm{~min}$, and has a flux emergence range of $\phi_{\mathrm{bp}}=4 \times 10^{16}-10^{19} \mathrm{Mx}$. Flux emergence is determined by the probability distribution of Thornton and Parnell (2011), giving an emergence rate of $1.2 \times 10^{-3} \mathrm{Mx} \mathrm{cm}^{-2} \mathrm{~s}^{-1}$. The smallest magnetic features that occur within this simulation have a flux of $10^{16} \mathrm{Mx}$. The magnetograms are all in flux balance and are periodic in the $x$ - and $y$-directions. For full details of the simulation, see Paper I. For the 3D simulations, we select a $48 \mathrm{hr}$ window of the synthetic magnetograms from the full $250 \mathrm{hr}$ set, as explained below, providing a series of 2881 synthetic magnetograms.

Figure 1(a) shows the total absolute flux for the $48 \mathrm{hr}$ section of the 2D simulation that is used for $3 \mathrm{D}$ modelling. The curve oscillates about a mean 
(a)

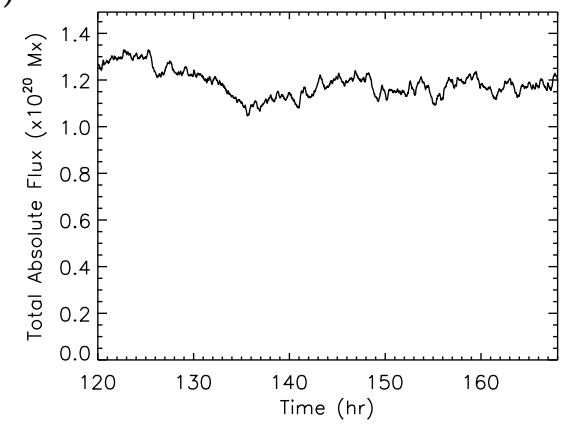

(b)

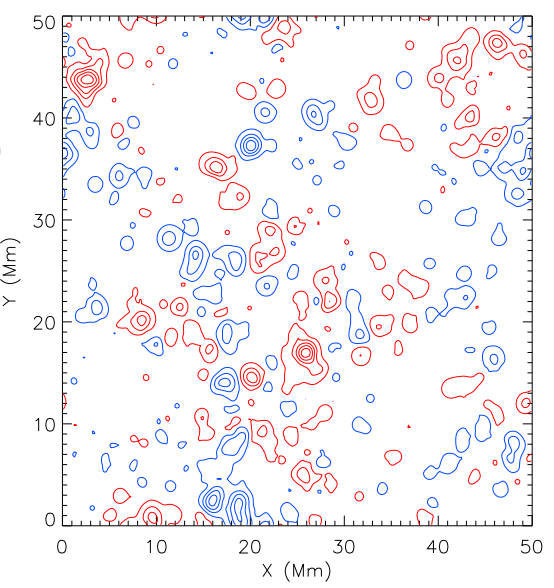

Figure 1. (a) Total absolute flux through the photosphere as a function of time for the 48 hr series of magnetograms used in our 3D models. (b) Synthetic magnetogram at $t=120$ hr, composed of 1499 individual magnetic elements. Ten contour levels of positive (red) and negative (blue) magnetic field are shown, with absolute values spaced evenly between $7.5 \mathrm{G}$ and $142.5 \mathrm{G}$.

value of $1.19 \times 10^{20} \mathrm{Mx}$, with a standard deviation of $5.79 \times 10^{18} \mathrm{Mx}$, or $4.9 \%$, indicating that the $2 \mathrm{D}$ simulation is in a steady state at this time.

Figures 1(b) shows an example of a synthetic magnetogram, taken at $t=120$ $\mathrm{hr}$, where red and blue contours represent positive and negative magnetic field, respectively. A movie showing the photospheric evolution of $B_{z}$ for the first $2 \mathrm{hr}$ is available (magnet48_bz_2hr.mpg). The magnetic elements are mainly located around the boundaries of the supergranules, forming the magnetic network (an image of the simulated supergranules can be seen in Figure 3(b) of Paper I). The supergranular flow profile is not time-evolving, so the general shape of the magnetic network does not vary much. Despite this limitation, the exact distribution of magnetic elements does change significantly throughout the 48 $\mathrm{hr}$ period. In Paper I it was determined that the photospheric recycle time for this series of synthetic magnetograms was $1.48 \mathrm{hr}$, in excellent agreement with Hagenaar, DeRosa, and Schrijver (2008)'s recycle time of $1-2 \mathrm{hr}$. Therefore the synthetic magnetogram series realistically simulates the dynamic nature of the magnetic carpet, making it a suitable lower boundary condition for 3D modelling.

\subsection{Coronal Magnetic Field Evolution}

We now discuss the set-up of the 3D model. We choose a numerical box of size $50 \times 50 \times 25 \mathrm{Mm}^{3}$, composed of $512 \times 512 \times 256$ grid cells. The box is periodic in the $x-$ and $y$-directions and closed at the top. Since magnetic flux may only enter and exit the box through the lower boundary, the synthetic magnetograms are required to be in complete flux balance. The periodicity of the side boundaries gives the effect of the region being surrounded by similar regions of small-scale magnetic carpet features. The initial condition for each simulation 
is a potential field extrapolated from the first magnetogram. We subsequently evolve the coronal magnetic field in response to photospheric boundary motions using a magnetofrictional relaxation technique (Yang, Sturrock, and Antiochos, 1986 Mackay and van Ballegooijen, 2006 : Yeates, Mackay, and van Ballegooijen, 2008 Mackay and van Ballegooijen, 2009: Mackay, Green, and van Ballegooijen, 2011. Meyer, Mackay, and van Ballegooijen, 2012). The magnetofrictional technique produces a continuous evolution of the coronal magnetic field, so that a 'memory' of flux connectivity and electric current systems is maintained from one step to the next. The coronal field induction equation is given by

$$
\frac{\partial \mathbf{A}}{\partial t}=\mathbf{v} \times \mathbf{B}+\boldsymbol{\epsilon}
$$

where $\mathbf{B}=\nabla \times \mathbf{A}$ is the magnetic field and $\mathbf{A}$ its the associated vector potential. The plasma velocity, $\mathbf{v}$, is given by

$$
\mathbf{v}=\frac{1}{\nu} \frac{\mathbf{j} \times \mathbf{B}}{B^{2}}
$$

where $\nu$ is the coefficient of friction, $\frac{1}{\nu}=8 \times 10^{4} \mathrm{~km}^{2} \mathrm{~s}^{-1}$, and $\mathbf{j}=\nabla \times \mathbf{B}$. This velocity describes the relaxation of the coronal magnetic field towards a non-linear force-free state in response to perturbations, and takes advantage of the fact that the Lorentz force, $\mathbf{j} \times \mathbf{B}$, is the dominant force within the corona. The term $\boldsymbol{\epsilon}$ within Equation 1 represents hyperdiffusion (e.g. Boozer (1986), van Ballegooijen and Cranmer (2008)) and is chosen to be of the form

$$
\boldsymbol{\epsilon}=\frac{\mathbf{B}}{B^{2}} \nabla \cdot\left(\eta_{4} B^{2} \nabla \alpha\right)
$$

where, $\eta_{4}=7.6 \times 10^{5} \mathrm{~km}^{4} \mathrm{~s}^{-1}$ and $\alpha$ is the scalar coefficient from the definition of a non-linear force-free field such that $\nabla \times \mathbf{B}=\alpha \mathbf{B}$. It is calculated as

$$
\alpha=\frac{\mathbf{j} \cdot \mathbf{B}}{B^{2}} \text {. }
$$

Hyperdiffusion aids the stability of the code by smoothing gradients in $\alpha$ and allows reconnection to occur. It acts to reduce the magnetic field to a linear force-free state, however the time scales of the present simulations are too short for such a state to be reached.

The evolution of the photospheric boundary perturbs the coronal field, which responds through Equations 1 and 2. This continual stressing and relaxing of the coronal field, in response to the lower boundary motions, evolves the field through a series of quasi-static, non-linear force-free equilibria.

\subsection{Lower Boundary Treatment}

The lower boundary treatment is the same as is described in Paper II, where a full description may be found. Since Equation 1 is specified in terms of the vector potential $\mathbf{A}$, the lower boundary is also required in terms of $\mathbf{A}$. A linear interpolation of the $A_{x}$ and $A_{y}$ corresponding to $B_{z}$ at $z=0 \mathrm{Mm}$ is carried 
out between each synthetic magnetogram. $B_{z}$ at $z=0 \mathrm{Mm}$ is analytically specified at each time step, rather than advected numerically (see Paper I). This avoids certain undesirable numerical effects such as numerical overshoot or pileup at cancellation sites. Between each analytical specification of the photospheric magnetic field, 500 interpolation steps are taken, corresponding to $0.12 \mathrm{~s}$ each. The magnetofrictional technique is applied during each of these interpolation steps so that the relaxation is gradual.

In total, four simulations are run, each with the same photospheric boundary evolution. Three of the simulations have an overlying, uniform magnetic field of strength $B_{0}=1 \mathrm{G}, 3 \mathrm{G}$ or $10 \mathrm{G}$, which points in the positive $x$-direction. The fourth simulation has no overlying field. For the cases with a uniform overlying field, we add this field to the initial potential field as follows: The potential field is computed in terms of the vector potential $\mathbf{A}$. We add the term $B_{0}\left(z_{\max }-z\right)$ to $A_{y}$ throughout the volume, where $B_{0}$ is the strength of the overlying field and $z$ is the height above the photosphere. Then, when we compute $\mathbf{B}=\nabla \times \mathbf{A}$, since the $x$-component is

$$
B_{x}=\frac{\partial A_{z}}{\partial y}-\frac{\partial A_{y}}{\partial z},
$$

this has the effect of adding a constant $B_{0}$ to $B_{x}$ throughout the volume. We also add a contribution $B_{0} z_{\max }$ to the $A_{y}$ component of all of the synthetic magnetograms that provide the lower boundary condition.

West et al. (2011) investigated an EIT wave during solar minimum and estimated the strength of the quiet Sun coronal magnetic field to be $0.7 \pm 0.7 \mathrm{G}$. A variety of authors have attempted to estimate the strength of active region coronal loops using coronal seismology, with values obtained anywhere in the range 3-90 G (Nakariakov and Ofman, 2001; Aschwanden et al., 2002, Verwichte et al., 2004, Van Doorsselaere et al., 2008; Wang, Inees, and Qiu, 2007), although De Moortel and Pascoe (2009) stress that coronal seismology does not necessarily reliably determine the strength of the magnetic field. Indeed, they find that when they apply the method of Nakariakov and Ofman (2001) to their 3D model of a coronal loop, it overestimates the strength of the magnetic field by $50 \%$. However, our no overlying field and $1 \mathrm{G}$ overlying field cases can be taken to represent the quiet Sun during solar minimum, while the $3 \mathrm{G}$ and $10 \mathrm{G}$ cases represent a quiet region of the Sun influenced by nearby active regions during solar maximum. The following sections give preliminary results of these 3D nonpotential simulations. In Section 3, we briefly discuss field line connectivity between magnetic elements. Section 3.1 focuses on the free magnetic energy, Section 3.2 the square of the current density $\left(j^{2}\right)$ and Section 3.3 on the energy dissipated. For both energies, we consider the time evolution of the quantity integrated over the whole volume, and also how it is affected by varying the overlying field strength. We then consider where the energy is located spatially.

\section{Results}

Figures 2(a) and (b) show images from the simulation with no overlying field, (c) and (d) the $3 \mathrm{G}$ overlying field simulation. The images on the left are both 

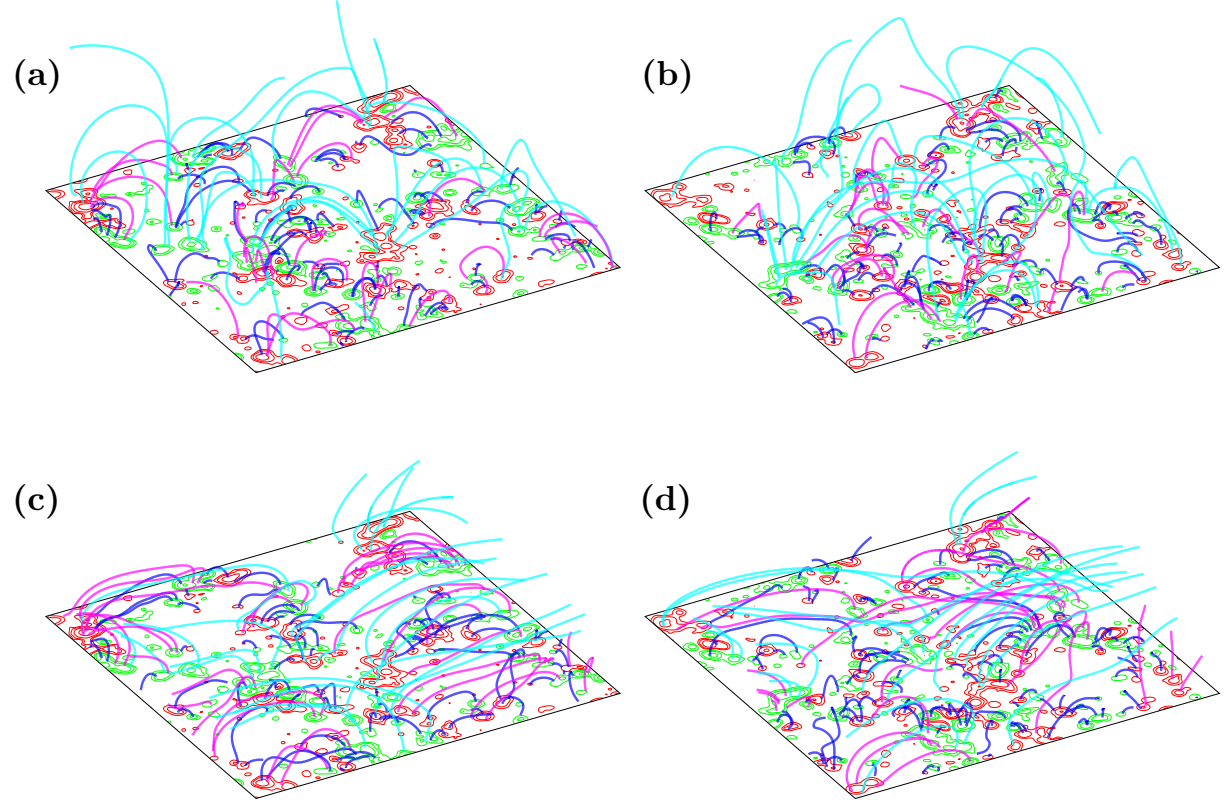

Figure 2. Coronal field images for the 3D simulation with (a) and (b) no overlying field, (c) and (d) a $3 \mathrm{G}$ overlying field. On the base, contours of positive (red) and negative (green) magnetic field are plotted. A selection of coronal magnetic field lines are shown in each case reaching maximum heights of $<2.5 \mathrm{Mm}$ (dark blue), $2.5-5 \mathrm{Mm}$ (magenta) and $>5 \mathrm{Mm}$ (pale blue). The images are taken at (a) and (c) $t=128 \mathrm{hr}$, (b) and (d) $t=168 \mathrm{hr}$.

taken at $t=128 \mathrm{hr}$ and the images on the right are shown at $t=168 \mathrm{hr}$. In all four images, contours of $B_{z}$ at $z=0 \mathrm{Mm}$ are plotted on the base (red=positive, green=negative). A selection of magnetic field lines are also over-plotted in each case, where dark blue, magenta and pale blue field lines reach a maximum height of $<2.5 \mathrm{Mm}, 2.5-5 \mathrm{Mm}$ and $>5 \mathrm{Mm}$ respectively. For comparison, field lines in Figures 2(c) and (d) are plotted from the same photospheric starting points as field lines in (a) and (b) respectively. As expected, we find that connections between magnetic elements within the simulation with no overlying field may reach much greater heights than those in the $3 \mathrm{G}$ simulation, as the overlying field suppresses the extension of the magnetic elements' field into the corona. Also, the connectivity is quite different between the images at $t=128 \mathrm{hr}$ and $t=168 \mathrm{hr}$, showing that the coronal field has changed significantly during this interval, as expected. The images here are intended only to give an indication of what the connectivity is like between the magnetic elements. A more in depth analysis will be carried out in future. 
(a)

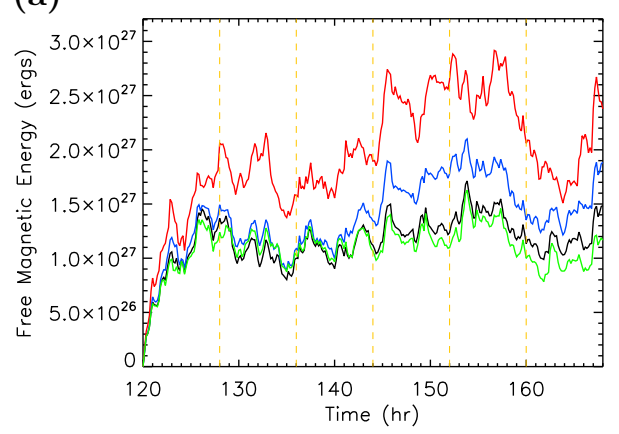

(c)

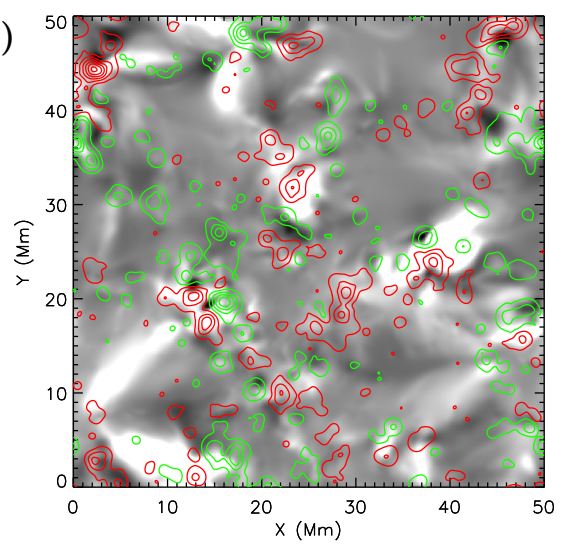

(e)

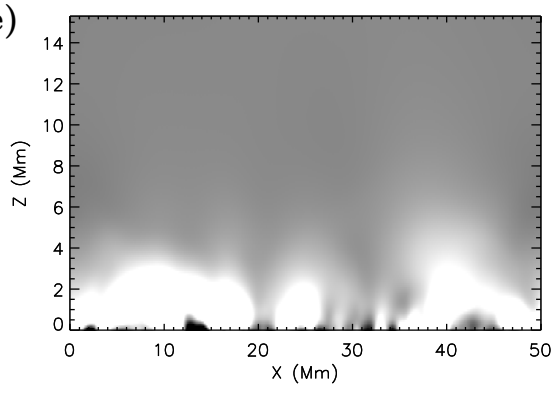

(b)

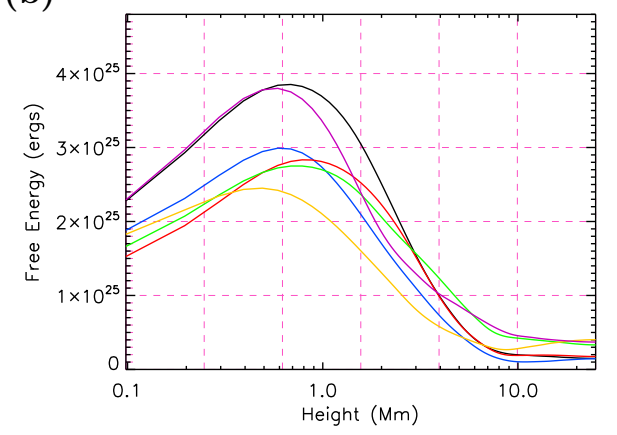

(d)

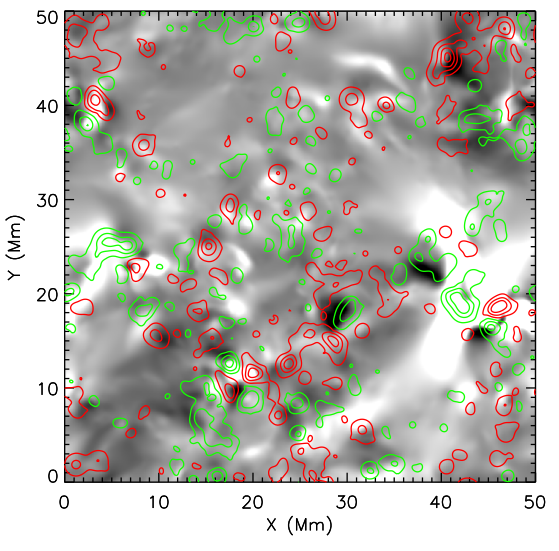

(f)

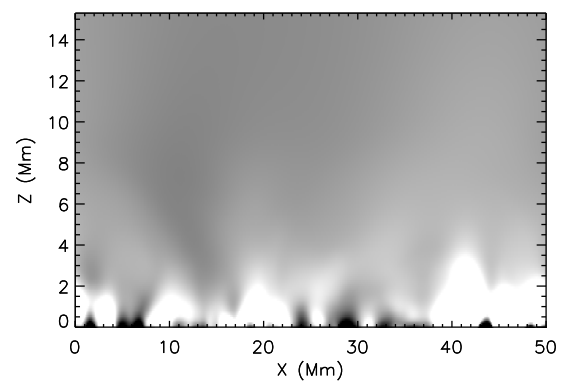

Figure 3. (a) Free magnetic energy as a function of time for the 3D simulations with no overlying field (green), $1 \mathrm{G}$ (black), $3 \mathrm{G}$ (blue) and $10 \mathrm{G}$ (red) overlying field. (b) Total free magnetic energy as a function of height for the simulation with a $3 \mathrm{G}$ overlying field. The curves show the free energy at $t=128 \mathrm{hr}$ (black), $t=136 \mathrm{hr}$ (blue), $t=144 \mathrm{hr}$ (red), $t=152 \mathrm{hr}$ (green), $t=160 \mathrm{hr}$ (yellow) and $t=168 \mathrm{hr}$ (purple). (c) and (d) Free magnetic energy density integrated along the LOS from above $(x-y$ plane), for the $3 \mathrm{G}$ overlying field simulation, at (c) $t=128 \mathrm{hr}$ and (d) $t=168 \mathrm{hr}$. White denotes regions where the free energy density is positive and black where it's negative, saturated at $\pm 1.9 \times 10^{22}$ ergs. Positive (red) and negative (green) contours of $B_{z}$ at $z=0 \mathrm{Mm}$ are over-plotted at levels of $\pm[7,13,27,53,106]$ G. (e) and (f) Free energy density integrated along the LOS, viewed from the side ( $x-z$ plane) saturated at $\pm 4.8 \times 10^{22} \mathrm{ergs}$, at (e) $t=128 \mathrm{hr}$ and (f) $t=168 \mathrm{hr}$. 
Table 1. Mean and maximum values of free magnetic energy for each simulation.

\begin{tabular}{ccc}
\hline $\begin{array}{c}\text { Simulation } \\
\left(B_{0}\right)\end{array}$ & $\begin{array}{c}\text { Mean Free Magnetic } \\
\text { Energy }\left(\times 10^{7} \mathrm{ergs} \mathrm{cm}^{-2}\right)\end{array}$ & $\begin{array}{c}\text { Maximum Free Magnetic } \\
\text { Energy }\left(\times 10^{7} \mathrm{ergs} \mathrm{cm}^{-2}\right)\end{array}$ \\
\hline $0 \mathrm{G}$ & 4.53 & 6.51 \\
$1 \mathrm{G}$ & 4.79 & 6.85 \\
$3 \mathrm{G}$ & 5.85 & 8.40 \\
$10 \mathrm{G}$ & 8.38 & 11.67 \\
\hline
\end{tabular}

\subsection{Free Magnetic Energy}

The presence of free magnetic energy within our 3D model is a significant difference to the potential field (minimum energy) models of the magnetic carpet coronal field discussed in the introduction. Figure 3(a) shows a plot of the free magnetic energy (ergs) as a function of time, defined as

$$
E_{\mathrm{f}}(t)=\int_{V} \frac{\left|\mathbf{B}_{\mathrm{nl}}\right|^{2}-\left|\mathbf{B}_{\mathrm{p}}\right|^{2}}{8 \pi} d V,
$$

where $\mathbf{B}_{\mathrm{nl}}$ is the non-linear force-free magnetic field and $\mathbf{B}_{\mathrm{p}}$ is the magnetic field of the corresponding potential field. Results are shown for the no overlying field (green), $1 \mathrm{G}$ (black), $3 \mathrm{G}$ (blue) and $10 \mathrm{G}$ (red) simulations. For each simulation, the free energy initially increases rapidly as the coronal field diverges from a potential state due to surface motions. For the no overlying field and $1 \mathrm{G}$ cases, the free energy then levels off and oscillates around a mean value of $1.13 \times 10^{27}$ ergs and $1.20 \times 10^{27} \mathrm{ergs}$, respectively, with standard deviations of $1.52 \times 10^{26}$ ergs and $1.74 \times 10^{26}$ ergs. For the $3 \mathrm{G}$ and $10 \mathrm{G}$ cases, the free energy also levels off to a lesser extent, but is less steady, with mean values of $1.46 \times 10^{27} \mathrm{ergs}$ and $2.10 \times 10^{27} \mathrm{ergs}$ and standard deviations of $2.84 \times 10^{26} \mathrm{ergs}$ and $4.06 \times 10^{26}$ ergs respectively. The mean and maximum values of free energy per unit area for each simulation are given in Table 1 . The trend is that a stronger overlying field leads to a greater build-up of free energy. In each case, the individual peaks in free energy differ between the simulations, however the general shape of the curve is the same for all four overlying field strengths. Therefore the overall behaviour of the free magnetic energy is largely dependent on the evolution of the photospheric magnetic field, rather than the strength of the overlying coronal field.

The variation of free energy with height is similar for all simulations, so here we just discuss the results from the $3 \mathrm{G}$ overlying field simulation. Figure $3(\mathrm{~b})$ shows plots of the free magnetic energy integrated over $x$ and $y$, as a function of height, for the simulation with a $3 \mathrm{G}$ overlying field. This is computed as follows:

$$
E_{\mathrm{f}}(z)=L_{z} \int_{y_{\min }}^{y_{\max }} \int_{x_{\min }}^{x_{\max }} \frac{\left|\mathbf{B}(x, y, z)_{\mathrm{nl}}\right|^{2}-\left|\mathbf{B}(x, y, z)_{\mathrm{p}}\right|^{2}}{8 \pi} d x d y,
$$


where $x_{\min }=y_{\min }=0 \mathrm{Mm}, x_{\max }=y_{\max }=50 \mathrm{Mm}$ and $L_{z}=0.098 \mathrm{Mm}$ is the length of a cell in $z$. The total free magnetic energy as a function of height is shown at times spaced evenly throughout the simulations, at $t=128 \mathrm{hr}$ (black), $t=136 \mathrm{hr}$ (blue), $t=144 \mathrm{hr}$ (red), $t=152 \mathrm{hr}$ (green), $t=160 \mathrm{hr}$ (yellow) and $t=168 \mathrm{hr}$ (purple). In Figure 3(a), which shows the total free magnetic energy as a function of time, the vertical yellow dashed lines are over-plotted on the graph at intervals of $8 \mathrm{hr}$, indicating the times at which the lines in Figure 3(b) are taken. In Figure 3(b), it can be seen that the ordering of the curves is not time dependent. Once the coronal field has evolved away from its initial potential state, the total amount of free magnetic energy within the volume depends upon how much is both built up and stored due to surface motions. For each of the curves in Figure 3(b), there is a peak between roughly $z=0.5 \mathrm{Mm}$ and $z=0.8$ $\mathrm{Mm}$, indicating that this is where the majority of the free magnetic energy is stored. The free energy then drops off rapidly after $z=1 \mathrm{Mm}$. As in Paper II, we find that the field departs most from a potential state low down in the corona, as this is close to where we are driving the evolution of the field by photospheric motions. In addition, most closed connections between magnetic elements (as opposed to connections from the magnetic elements to the overlying field) are found to be low lying (e.g. Priest, Heyvaerts, and Title (2002), Close et al. $(2003)$ ), and it is along these connections that free energy is stored. A movie showing the time evolution of the free magnetic energy as a function of height for the $3 \mathrm{G}$ simulation is available (magnet48b_free_ht.mpg). The movie shows that the free energy is highly dynamic and rapidly evolving. The height of the curve is continually changing, however it can be seen that the peak in the curve tends to remain between $z=0.5 \mathrm{Mm}$ and $z=0.8 \mathrm{Mm}$.

Figures $3(\mathrm{c})$ and (d) show the free magnetic energy density, $\frac{B_{\mathrm{nl}}^{2}-B_{\mathrm{p}}^{2}}{8 \pi}$, integrated in $z$, in the $x-y$ plane for the $3 \mathrm{G}$ simulation at $t=128 \mathrm{hr}$ and $t=168 \mathrm{hr}$, respectively. This is computed as follows:

$$
E_{\mathrm{f}}(x, y)=A \int_{z_{\min }}^{z_{\max }} \frac{\left|\mathbf{B}(x, y, z)_{\mathrm{nl}}\right|^{2}-\left|\mathbf{B}(x, y, z)_{\mathrm{p}}\right|^{2}}{8 \pi} d z,
$$

where $A=L_{x} L_{y}, L_{x}=0.098 \mathrm{Mm}$ is the length of a cell in $x$ and $L_{y}=0.098$ $\mathrm{Mm}$ is the length of a cell in $y$. White patches indicate where the free energy density is positive, i.e. where $B_{\mathrm{nl}}^{2}>B_{\mathrm{p}}^{2}$, black where the free energy density is negative, $B_{\mathrm{nl}}^{2}<B_{\mathrm{p}}^{2}$. Note that the total free magnetic energy integrated over the volume is always positive (see Figure $3(\mathrm{a})$ ). Positive (red) and negative (green) contours of $B_{z}$ at $z=0 \mathrm{Mm}$ are over-plotted. We define free magnetic energy to be 'stored' at locations where the line-of-sight (LOS) integrated free magnetic energy density is positive. From these images, it can be seen that free magnetic energy may be stored both at the boundaries between supergranular cells and within the cells themselves.

In Paper II, it was found that when the evolution of the magnetic elements disturbed a larger volume of the overlying field, a greater amount of free energy was built up. It was also found that closed connections between the magnetic elements are required, along which the free energy may be stored. In agreement with Paper II, we find that free energy is stored mainly in two locations. Firstly, 
we see that white patches are located around supergranule cell boundaries where the magnetic network is formed. Large numbers of magnetic elements are swept to these locations by supergranular flows, and continually interact with one another, cancelling, coalescing and fragmenting. This continual evolution of the magnetic elements results in a large build up of free energy, which may then be stored along the multiple connections that form between nearby magnetic elements that lie in the network. The second location where we see free energy stored is along long-lived, far-reaching, twisted connections between magnetic elements. Such connections may stretch across supergranule cells, between magnetic elements located at opposite boundaries (examples of this will be given later, in Figure 4. Longer connections will clearly disturb a larger volume of the surrounding coronal magnetic field, hence building up free energy. A movie showing the free magnetic energy density, integrated in $z$, in the $x-y$ plane is available (magnet48b_free_xy_bz.mpg), with contours of $B_{z}$ at $z=0 \mathrm{Mm}$ overplotted. Within the movie, one can see that regions of positive free energy density are continually evolving in response to photospheric motions. In particular, occasional large patches of positive free energy density develop around the magnetic network. One can also see long-lived bands of positive free magnetic energy density stretching across supergranules, between magnetic elements. Many of the regions of free magnetic energy density last for several hours. For readers unable to view the movie, six still images spaced $1 \mathrm{hr}$ apart are included in Appendix A (Figure 8), to give an impression of the time-scale of the evolution.

Figures 3(e) and (f) show $x-z$ plane images of the LOS integrated free magnetic energy density, saturated at $\pm 4.8 \times 10^{22}$ ergs, for the $3 \mathrm{G}$ simulation at $t=128 \mathrm{hr}$ and $t=168 \mathrm{hr}$, respectively. A movie of the free magnetic energy density in the $x-z$ plane, integrated in $y$, is available (magnet48b_free_xz.mpg). In both the movie and the still images, it can be seen that the free energy is mainly located low down, with the bulk of it being below $z=3 \mathrm{Mm}$. This is where many closed connections exist between the magnetic elements and a larger departure of the magnetic field from a potential state is found. One can see that the locations of positive free energy density are highly dynamic and there exist long-lived 'bulbs' of positive free energy density, where it is stored within the corona along closed connections between magnetic elements. Similar evolution of the LOS integrated free magnetic energy density is seen in the $y-z$ plane and for different strengths of overlying field.

Figures 4(a) and (b) show two zoomed in sections of the $x-y$ plane images of the LOS integrated free energy density taken at $t=128 \mathrm{hr}$ and $t=168$ hr, respectively, from Figures 3(c) and (d). A selection of closed field lines has been over-plotted in blue in each case. Figure 4(a) shows the band of positive free energy density that can be seen lying across the lower left supergranule in Figure 3(c), while Figure 4(b) shows the band of positive free energy density across the lower right supergranule in Figure 3(d). In both zoomed images, twisted magnetic field lines connect between various magnetic elements on either side of the supergranule. The free energy is stored in these regions of complex connections. Figure 4(c) shows an $x-z$ plane image of free energy density integrated in $y$ at $t=128 \mathrm{hr}$, and is a side view of the band of positive free energy density in Figure 4(a). Similarly, Figure 4(d) is a side view of the band of 

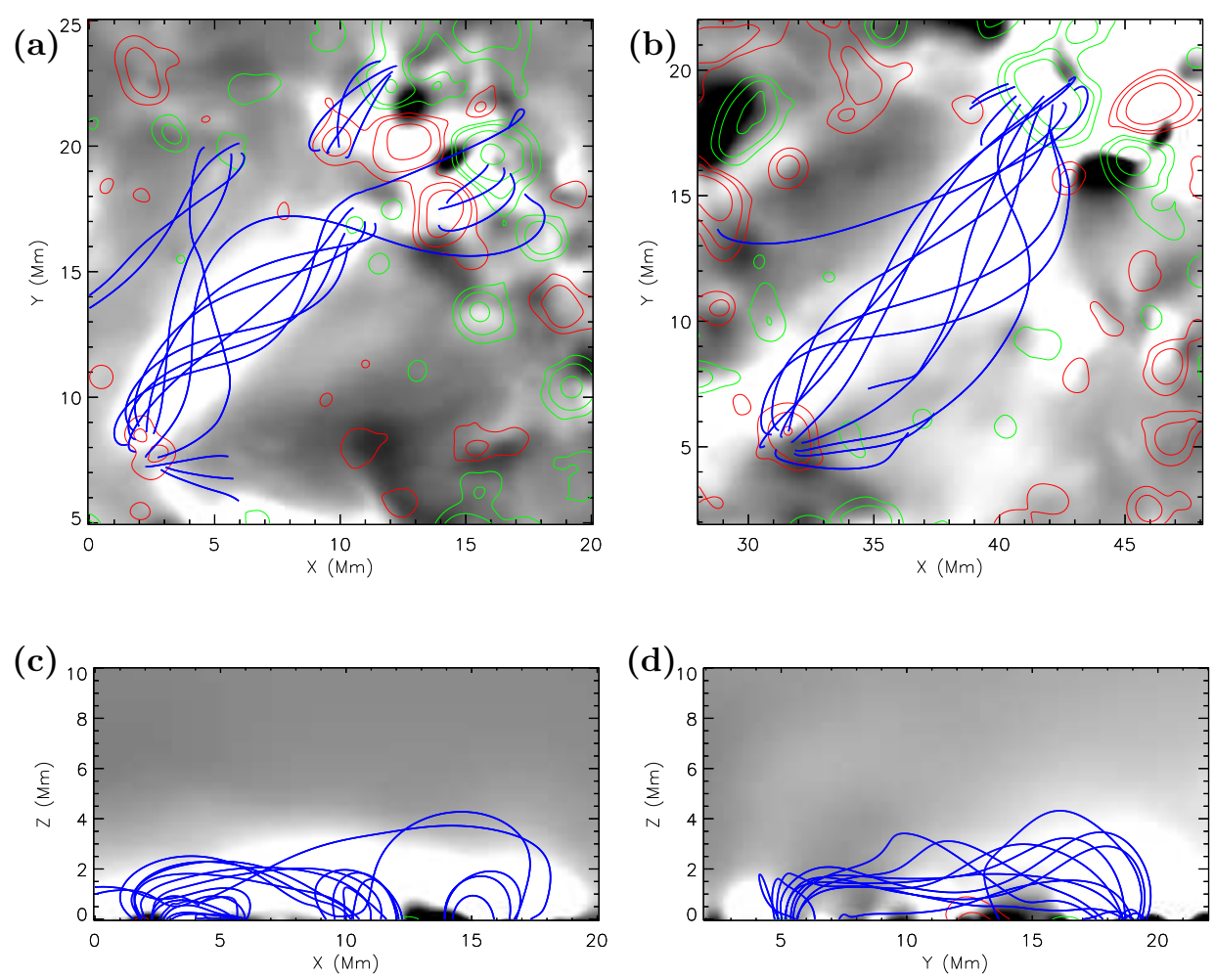

Figure 4. All images are for the $3 \mathrm{G}$ simulation. (a) and (b) Free magnetic energy density integrated in $z$, shown in the $x-y$ plane. The images are white in regions where the free energy density is positive, black where the free energy density is negative. Positive (red) and negative (green) contours of $B_{z}$ at $z=0 \mathrm{Mm}$ are over-plotted at levels of $\pm[7,13,27,53,106] \mathrm{G}$. The images are shown at (a) $t=128 \mathrm{hr}$ and (b) $t=168 \mathrm{hr}$. (c) Free magnetic energy integrated in $y$, shown in the $x-z$ plane at $t=128 \mathrm{hr}$. (d) Free magnetic energy density integrated in $x$, shown in the $y-z$ plane at $t=168 \mathrm{hr}$. A selection of field lines are over-plotted in blue on each image.

positive free energy density in Figure 4 (b) and shows a $y-z$ plane image of free energy density integrated in $x$ at $t=168 \mathrm{hr}$. For each of the cases, a complex structure of the magnetic field can be seen.

\subsection{Current Density}

The square of the current density, $j^{2}$, is of interest because it indicates locations of possible Ohmic heating, $\frac{j^{2}}{\sigma}$. However, it should be noted that in the present simulations, due to the simplified form used, we do not have an energy equation. Figures 5(a) and (b) show images in the $x-y$ plane of $j^{2}$ integrated in $z$, at $t=128 \mathrm{hr}$ and $t=168 \mathrm{hr}$, respectively. This is computed as follows:

$$
E_{j}(x, y)=\int_{z_{\min }}^{z_{\max }} j(x, y, z)^{2} d V .
$$


(a)

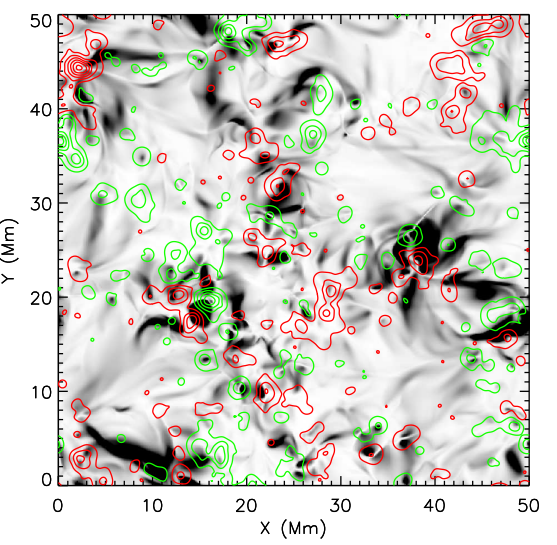

(c)

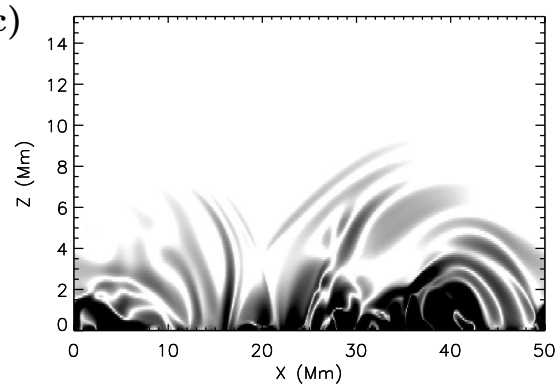

(e)

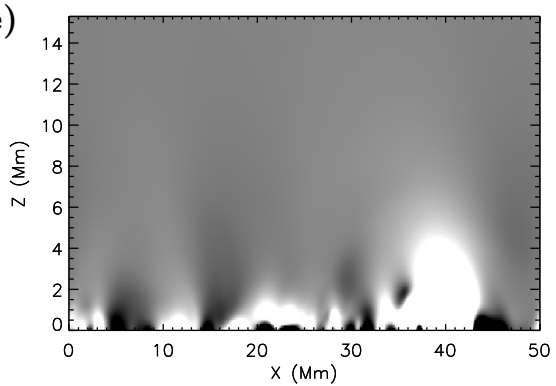

(b)

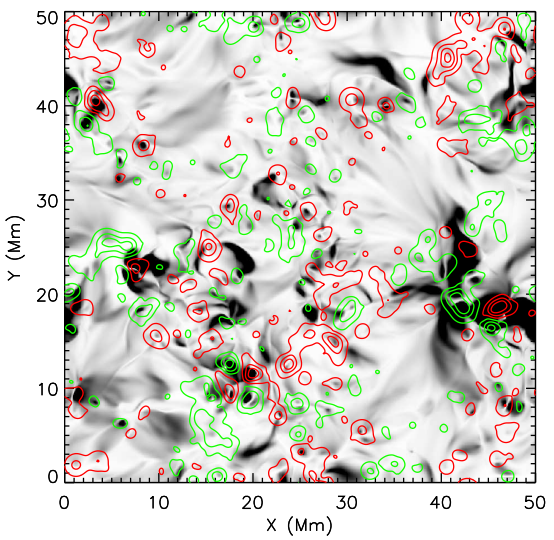

(d)

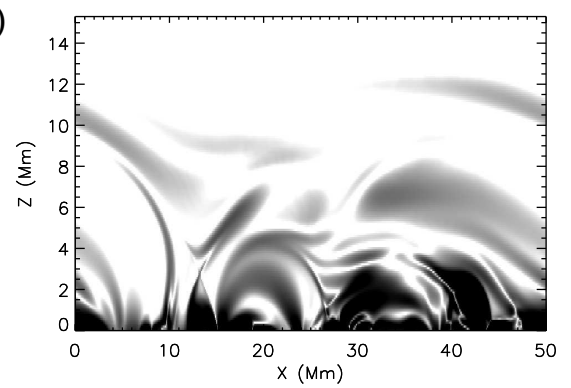

(f)

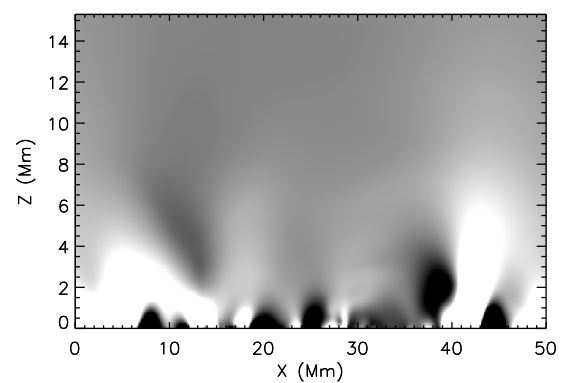

Figure 5. All images are for the $3 \mathrm{G}$ simulation. For (a)-(d), darker regions correspond to higher values. (a) and (b) Normalised $j^{2}$ integrated in $z$, shown in the $x-y$ plane. Contours of positive (red) and negative (green) magnetic field at $z=0 \mathrm{Mm}$ are over-plotted, at levels of $\pm[7,13,27,53,106] \mathrm{G}$. (c) and (d) logarithm of normalised $j^{2}$ in the $x-z$ plane at $y=25 \mathrm{Mm}$. (e) and (f) free magnetic energy density shown in the $x-z$ plane at $y=25 \mathrm{Mm}$, saturated at $\pm 1.9 \times 10^{20}$ ergs. Images in the left-hand column are shown at $t=128 \mathrm{hr}$ and in the right-hand column at $t=168 \mathrm{hr}$.

Note that the colour table has been reversed, so that darker regions correspond to higher values of $E_{j}(x, y)$. Contours of $B_{z}$ at $z=0 \mathrm{Mm}$ are over-plotted at the same levels as in Figures 3(c) and (d). On comparison with the $x-y$ plane images 
of free magnetic energy in Figures 3 (c) and (d), the locations of high $j^{2}$ and of positive free magnetic energy density seem to match very well. The regions of high $j^{2}$ appear to be strongest in the magnetic network. Within a force-free field, by definition, $\mathbf{j} \times \mathbf{B}=0$, hence $\mathbf{j}$ is parallel to $\mathbf{B}$. This means that we can express $\mathbf{j}$ as a scalar multiple of $\mathbf{B}, \mathbf{j}=\alpha \mathbf{B}$, where $\alpha$ is a scalar representing the twist of the magnetic field with respect to the corresponding potential field. Therefore, it makes sense that $j^{2}$ is at its largest near the magnetic sources, where $\mathbf{B}$ is largest. We also see fainter bands of $j^{2}$ stretching across supergranules, often in the same places as bands of positive free magnetic energy density. Again, it makes sense for $j^{2}$ to be high in such locations as free magnetic energy is built up in regions of high non-potentiality (large $|\alpha|$ ) which arise due to non-zero $\mathbf{j}$ $(=\alpha \mathbf{B})$. The evolution of $j^{2}$, integrated in $z$, in the $x-y$ plane can be seen in the movie, magnet48b_j_xy_bz.mpg, for the $3 \mathrm{G}$ simulation. Six still images from this movie, spaced $1 \mathrm{hr}$ apart, are included in Appendix A (Figure 9), to give an impression of the evolution for those who cannot view the movie. Although regions of $j^{2}$ are often co-located with regions of positive free energy density, the regions of $j^{2}$ appear to be more rapidly evolving than those of free energy. The spatial distribution of $j^{2}$ is quite different from one hour to the next.

Figures 5(c) and (d) show images of the logarithm of $j^{2}$ in the $x-z$ plane at $y=25 \mathrm{Mm}$, at $t=128 \mathrm{hr}$ and $t=168 \mathrm{hr}$, respectively. The regions of $j^{2}$ appear to be well structured, and obviously follow the shape of the magnetic field (as $\mathbf{j}$ is parallel to B). Figures 5(e) and (f) show images of the free magnetic energy density at $y=25 \mathrm{Mm}$, shown at the same times as (c) and (d) respectively. It can be seen that regions of positive free energy density tend to be co-located with regions of high $j^{2}$.

\subsection{Energy Dissipated}

In addition to the free magnetic energy stored within the system, we consider energy that is being continually dissipated due to the relaxation processes within the model applied. This is described by the energy dissipation term,

$$
Q=Q_{\mathrm{frc}}+Q_{\mathrm{hd}}=\frac{B^{2}}{4 \pi} \nu|\mathbf{v}|^{2}+\frac{B^{2}}{4 \pi} \eta_{4}|\nabla \alpha|^{2} .
$$

The first term, $Q_{\mathrm{frc}}$, represents energy dissipation due to magnetofriction, which is released as the coronal magnetic field relaxes towards a force-free state (Yang, Sturrock, and Antiochos, 1986). The second term, $Q_{\text {hd }}$, represents energy dissipation due to hyperdiffusion (Boozer, 1986). It has previously been interpreted as the rate at which magnetic energy is converted into heat during the relaxation of the magnetic field (van Ballegooijen and Cranmer, 2008). For the derivation of $Q$ and a full description of the terms $Q_{\text {frc }}$ and $Q_{\mathrm{hd}}$, see Paper II.

Figure 6(a) shows a plot of the rate of energy dissipation per unit area, due to magnetofriction, $\frac{1}{S} \int_{V} Q_{\text {frc }} d V$, as a function of time, for the no overlying field (green), $1 \mathrm{G}$ (black), $3 \mathrm{G}$ (blue) and $10 \mathrm{G}$ (red) simulations. $S=2.5 \times 10^{19}$ $\mathrm{cm}^{2}$ is the area of the photospheric boundary surface. It can be seen that $Q_{\mathrm{frc}}$ is not strongly dependent on the overlying field strength. A stronger overlying field leads to slightly higher $Q_{\mathrm{frc}}$, but the variation of values of $Q_{\mathrm{frc}}$ within each curve is 
(a)

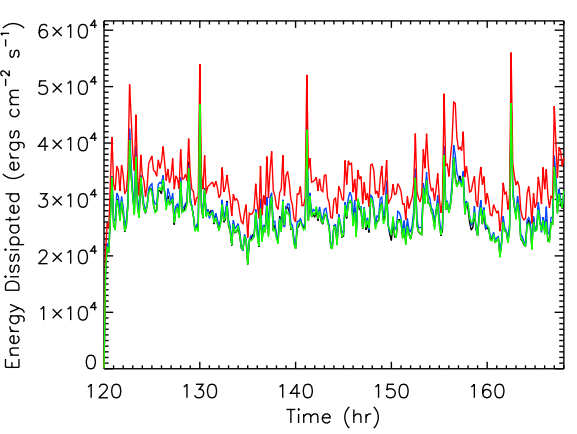

(c)

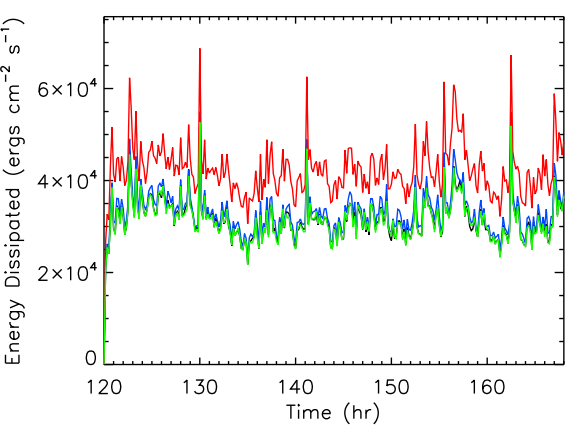

(e)

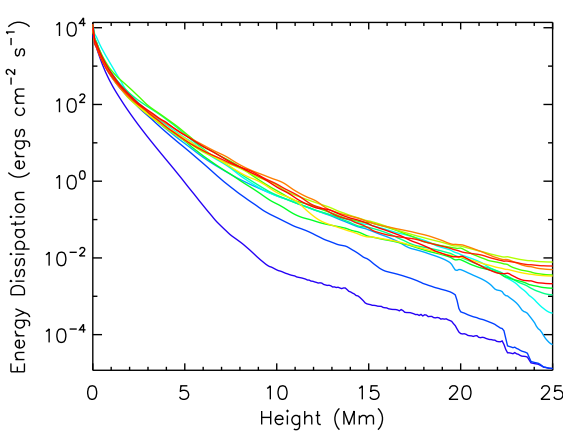

(b)

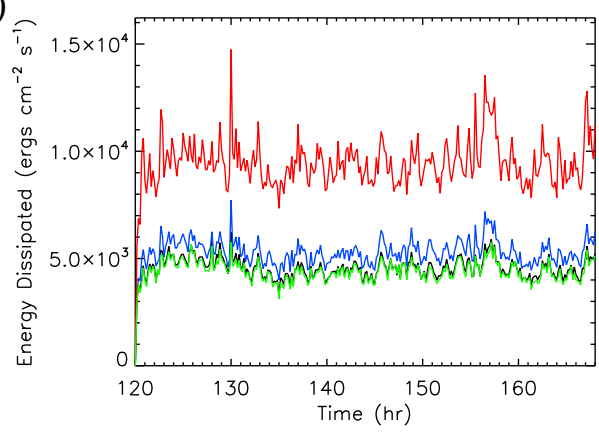

(d)

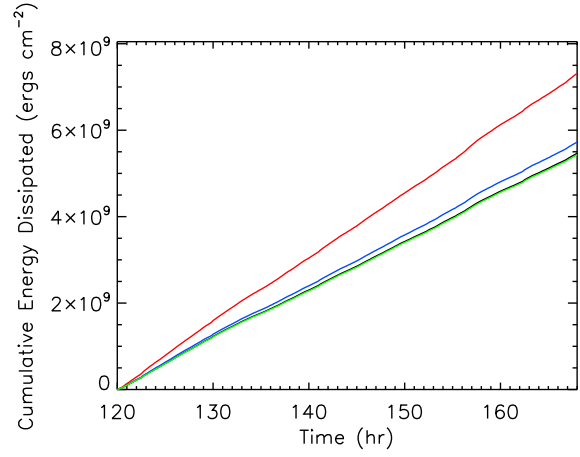

(f)

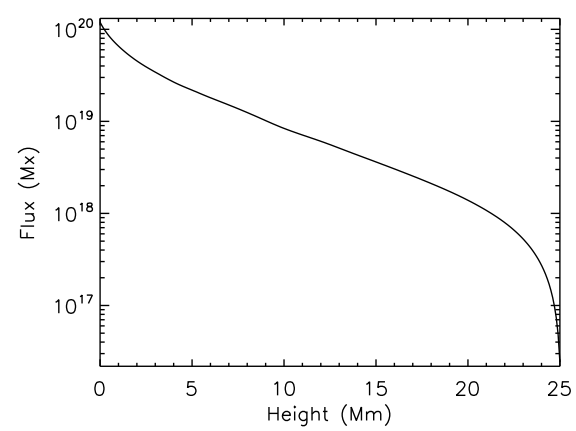

Figure 6. (a) - (d) Energy dissipated per unit area as a function of time for the 3D simulations with no overlying field (green), $1 \mathrm{G}$ (black), $3 \mathrm{G}$ (blue) and $10 \mathrm{G}$ (red) overlying field. (a) Rate of energy dissipation due to magnetofriction, $\frac{1}{S} \int_{V} Q_{\mathrm{frc}} d V$, (b) rate of energy dissipation due to hyperdiffusion, $\frac{1}{S} \int_{V} Q_{\mathrm{hd}} d V$, and (c) total rate of energy dissipation, $E_{\mathrm{q}}(t)$. (d) Cumulative energy dissipated as a function of time, $E_{\mathrm{d}}(t)$. (e) Rate of energy dissipation (integrated over $x$ and $y$ ) as a function of height for the $3 \mathrm{G}$ simulation. Six curves are plotted at $50 \mathrm{~min}$ intervals from $t=120.17-124.33 \mathrm{hr}$, the remaining six curves are plotted at $8 \mathrm{hr}$ intervals from $t=128-168 \mathrm{hr}$. Colours span from blue to red with increasing time. (f) Total absolute flux through surfaces of $z=$ constant, as a function of height, at $t=168 \mathrm{hr}$.

much larger than the variation of values between the curves for different overlying field strengths. Table 2 shows the mean and maximum values of $\frac{1}{S} \int_{V} Q_{\mathrm{frc}} d V$ for each overlying field strength simulation. The difference in mean values between 
Table 2. Mean and maximum values of $Q_{\mathrm{frc}}, Q_{\mathrm{hd}}$ and $Q$ integrated over the volume $\left(E_{\mathrm{q}}(t)\right)$, and cumulative energy dissipated for each simulation. Typical horizontal magnetic field, $B_{h}$, and Poynting flux order of magnitude estimate, $P_{1} / S$, for each simulation.

\begin{tabular}{|c|c|c|c|c|c|}
\hline & $B_{0}$ & $0 \mathrm{G}$ & $1 \mathrm{G}$ & $3 \mathrm{G}$ & $10 \mathrm{G}$ \\
\hline$\frac{1}{S} \int_{V} Q_{\mathrm{frc}} d V$ & Mean & 2.66 & 2.67 & 2.74 & 3.25 \\
\hline$\left(\times 10^{4}\right.$ ergs $\left.\mathrm{cm}^{-2} \mathrm{~s}^{-1}\right)$ & $\operatorname{Max}$ & 4.71 & 4.78 & 4.91 & 5.60 \\
\hline$\frac{1}{S} \int_{V} Q_{\mathrm{hd}} d V$ & Mean & 0.44 & 0.45 & 0.53 & 0.95 \\
\hline$\left(\times 10^{4} \mathrm{ergs} \mathrm{cm}^{-2} \mathrm{~s}^{-1}\right)$ & $\operatorname{Max}$ & 0.58 & 0.62 & 0.77 & 1.47 \\
\hline$E_{\mathrm{q}}(t)$ & Mean & 3.10 & 3.12 & 3.27 & 4.20 \\
\hline$\left(\times 10^{4}\right.$ ergs $\left.\mathrm{cm}^{-2} \mathrm{~s}^{-1}\right)$ & $\operatorname{Max}$ & 5.26 & 5.30 & 5.49 & 6.87 \\
\hline Typical $B_{h}(\mathrm{G})$ & & 6.5 & 6.6 & 7.2 & 12.1 \\
\hline$P_{1} / S\left(\times 10^{4}\right.$ ergs cm $\left.^{-2} \mathrm{~s}^{-1}\right)$ & & 5.96 & 6.05 & 6.60 & 11.09 \\
\hline$S E_{\mathrm{d}}\left(t_{\max }\right)\left(\times 10^{29} \mathrm{ergs}\right)$ & & 1.35 & 1.37 & 1.43 & 1.83 \\
\hline$E_{\mathrm{d}}\left(t_{\max }\right)\left(\times 10^{9}\right.$ ergs $\left.\mathrm{cm}^{-2}\right)$ & & 5.43 & 5.47 & 5.73 & 7.31 \\
\hline
\end{tabular}

the $0 \mathrm{G}$ and $10 \mathrm{G}$ cases is just $0.59 \times 10^{4} \mathrm{ergs}_{\mathrm{cm}}^{-2} \mathrm{~s}^{-1}$. However, from the plot in Figure 6(a), each curve has a variation of around $2.9-3.3 \times 10^{4} \mathrm{ergs}$ $\mathrm{cm}^{-2} \mathrm{~s}^{-1}$ between its maximum and minimum. Therefore, the energy dissipation due to magnetofriction is predominantly dependent upon the evolution of the photospheric magnetic field driving change within the coronal field.

Figure 6(b) shows the rate of energy dissipation per unit area due to hyperdiffusion, $\frac{1}{S} \int_{V} Q_{\mathrm{hd}} d V$, as a function of time, where lines are coloured as in Figure 6(a). Very little difference can be seen between the curves for the no overlying field and $1 \mathrm{G}$ cases. The $3 \mathrm{G}$ case results in slightly higher $Q_{\mathrm{hd}}$, while the $10 \mathrm{G}$ case results in significantly higher $Q_{\mathrm{hd}}$. Also, for the $10 \mathrm{G}$ case there is a larger variation in the values of $Q_{\mathrm{hd}}$ than in the no overlying field or $1 \mathrm{G}$ cases. Thus, $Q_{\text {hd }}$ is clearly dependent on the strength of the overlying field. While this is the case, the general shape of all of the curves are the same, implying that like $Q_{\mathrm{frc}}, Q_{\mathrm{hd}}$ is also predominantly dependent on the evolution of the photospheric magnetic field driving changes within the coronal volume. The mean and maximum values for $\frac{1}{S} \int_{V} Q_{\mathrm{hd}} d V$ for each strength of overlying field are given in Table 2, For each case, the mean values for $Q_{\mathrm{hd}}$ are $3.4-6.0$ times smaller than the mean values for $Q_{\mathrm{frc}}$, and the maximum values for $Q_{\mathrm{hd}}$ are $3.8-8.1$ times smaller than the maximum values for $Q_{\mathrm{frc}}$.

Figure 6(c) shows a plot of the total rate of energy dissipation per unit area, $E_{\mathrm{q}}(t)=\frac{1}{S} \int_{V} Q d V$, as a function of time, with lines coloured as in Figure 6(a). Since throughout each simulation, $Q_{\text {frc }}$ is larger than $Q_{\mathrm{hd}}$, the curves for $Q$ follow the same trend as those for $Q_{\mathrm{frc}}$. A stronger overlying field leads to slightly higher $Q$, but the variation of $Q$ within each curve $\left(3.1-3.8 \times 10^{4} \mathrm{ergs} \mathrm{cm}^{-2} \mathrm{~s}^{-1}\right)$ is larger than the variation between the simulations with different overlying field 
strengths $\left(1.1 \times 10^{4} \mathrm{ergs} \mathrm{cm}^{-2} \mathrm{~s}^{-1}\right.$ difference between the mean values for the no overlying field and $10 \mathrm{G}$ cases). Therefore, the overall energy dissipation is determined mainly by the photospheric evolution of the magnetic field. The mean and maximum values of $E_{\mathrm{q}}(t)$ for each simulation are given in Table 2. Figure 6(d) shows the cumulative energy dissipated, per unit area, as a function of time, $E_{\mathrm{d}}(t)$, for each strength of overlying field, obtained by integrating $Q$ over both the volume and time:

$$
E_{\mathrm{d}}(t)=\frac{1}{S} \int_{0}^{t}\left[\int_{V} Q d V\right] d t
$$

We see that a stronger overlying field leads to a greater cumulative amount of energy dissipated. The slopes of these curves are given by the mean values of $E_{\mathrm{q}}(t)$ in Table 2, and can be related to the overlying field strength $\left(B_{0}\right)$ by a quadratic:

$$
\overline{E_{\mathrm{q}}(t)}=75.6 B_{0}^{2}+339.8 B_{0}+3.1 \times 10^{4} .
$$

The values for the total energy dissipated by the end of each simulation $\left(t=t_{\max }\right)$ are also given in Table 2 .

The rate of energy dissipation per unit area $\left(\mathrm{ergs} \mathrm{cm}^{-2} \mathrm{~s}^{-1}\right)$ in each simulation is on average $3.1-4.2 \times 10^{4} \mathrm{ergs} \mathrm{cm}^{-2} \mathrm{~s}^{-1}$. These values are too low to explain the radiative losses of the quiet Sun corona, being only $31-42 \%$ of the $10^{5} \mathrm{ergs} \mathrm{cm}^{-2}$ $\mathrm{s}^{-1}$ calculated by Withbroe and Noyes (1977) and $6.3-8.6 \%$ of the $4.9 \times 10^{5} \mathrm{ergs}$ $\mathrm{cm}^{-2} \mathrm{~s}^{-1}$ calculated by Habbal and Grace (1991). Due to the fact that the values are too low, it is of interest to compare the energy dissipated to the Poynting flux through the photospheric boundary, as clearly we cannot dissipate more energy than has been injected. Considering the plots of free magnetic energy as a function of time (Figure 3(a)), they are roughly steady, at least for the $1 \mathrm{G}, 3$ $\mathrm{G}$ and no overlying field cases. This implies that the energy dissipated $\left(E_{\mathrm{q}}(t)\right)$ should roughly balance the Poynting flux injected. The Poynting flux through the photospheric boundary is given by

$$
P=-\frac{c}{4 \pi} \int_{S}(\mathbf{E} \times \mathbf{B}) \cdot d \mathbf{S}
$$

where $d \mathbf{S}=d S \hat{\mathbf{n}}$ and $S$ is the area of the photospheric boundary surface with unit normal vector $\hat{\mathbf{n}}$. From Ohm's law,

$$
\mathbf{E}=-\frac{1}{c} \mathbf{v} \times \mathbf{B}
$$

so Equation 9 simplifies to

$$
P=\frac{1}{4 \pi} \int_{S}\left[v_{x} B_{x} B_{z}+v_{y} B_{y} B_{z}-v_{z} B_{x}^{2}-v_{z} B_{y}^{2}\right] d S .
$$

The individual terms in Equation 11 may be split into two distinct groups. Those involving $v_{x}$ and $v_{y}$ represent energy flow into or out of the domain due to horizontal boundary flows. The terms involving $v_{z}$ represent energy flow into or out of the domain due to flux emergence or cancellation. 
As computed in Mackay, Green, and van Ballegooijen (2011), an order of magnitude estimate of the Poynting flux due to horizontal boundary flows is:

$$
P_{1} \approx \frac{v_{h} B_{z} B_{h} S}{4 \pi}
$$

where $B_{h}$ and $B_{z}$ are typical values for horizontal and vertical magnetic field at the photosphere and $v_{h}$ is a typical value for horizontal velocities at the photosphere.

In all simulations, the mean vertical field strength is $B_{z}=4.8 \mathrm{G}$ over a photospheric area of $S=2.5 \times 10^{19} \mathrm{~cm}^{2}$ and the mean value of our supergranular velocity profile at the photosphere is $v_{h}=0.24 \mathrm{~km} \mathrm{~s}^{-1}$. The only other parameter is the typical value for the horizontal field strength, $B_{h}$, which varies from one simulation to the next due to the overlying field. The mean values of $B_{h}$ for each simulation are listed in Table 2 along with the mean energy dissipation, $E_{\mathrm{q}}(t)$, and the value of $P_{1}$ calculated for each simulation. The values in Table 2 show that our order of magnitude estimate for energy injected due to horizontal flows is within a factor of $2-3$ of the energy dissipated for each simulation, so are in good agreement.

While we are able to estimate the Poynting flux resulting from horizontal flows, we cannot calculate the Poynting flux from vertical flows as, due to our special boundary treatment (Section 2.2), there is no $v_{z}$ defined on the photospheric boundary of our model. The component of the Poynting flux due to $v_{z}$ would likely lead to both injection and removal of energy respectively during emergence and cancellation events. As the flux domain is in a steady state, with the rates of flux emergence and cancellation roughly identical (see Paper I), we may assume that these processes lead to no net injection of energy. In addition, Parnell and De Moortel (2012) have estimated, using observational data, that the Poynting flux injected due to the emergence of magnetic flux is significantly smaller than that injected due to the horizontal motions of existing magnetic flux.

Assuming equal rates of energy input and loss due to emergence and cancellation, this calculation shows that the energy injected does in fact match the energy dissipated within an order of magnitude. This implies that the reason why the energy dissipated within our simulations is not high enough to account for coronal radiative losses is in part due to the fact that not enough energy has been injected in the first place. Therefore, in future simulations, increasing $v_{h}$, $B_{h}$ or $B_{z}$ may lead to an increase in energy dissipation.

We note that other possible reasons for the energy dissipation rate being too low are that this is not a true physical dissipation, and many simplifications have been made for our model. For example, our model contains no plasma and we do not resolve wave motions. The relative simplicity of the synthetic magnetograms compared to observed magnetograms may also be a factor. While the values calculated for $E_{\mathrm{q}}(t)$ are too low to explain coronal heating, it is of interest for us to consider the location and structure of the energy dissipation term, $Q$.

Figures 6(a)-(d) consider the volume integrated rate of energy dissipation over the entire 3D simulation for each strength of overlying field. We now consider where $Q$ is spatially located within individual frames of the $3 \mathrm{G}$ simulation. 
Although results are presented here only for the $3 \mathrm{G}$ simulation, similar results are found for other strengths of the overlying field. Figure 6(e) shows the rate of energy dissipation (integrated in $x$ and $y$ ) as a function of height. This is computed with units of ergs $\mathrm{cm}^{-2} \mathrm{~s}^{-1}$ as follows:

$$
E_{\mathrm{q}}(z)=\frac{L_{z}}{S} \int_{y_{\min }}^{y_{\max }} \int_{x_{\min }}^{x_{\max }} Q(x, y, z) d x d y .
$$

Six curves are plotted at intervals of $50 \mathrm{~min}$ from $t=120.17-124.33 \mathrm{hr}$, the other six are plotted at intervals of $8 \mathrm{hr}$ from $t=128-168 \mathrm{hr}$. Colours span from blue to red with increasing time. The curve at the earliest time $(t=120.17 \mathrm{hr})$ is lowest, as the coronal magnetic field is still close to potential. The height of the curves increases with increasing time until a near steady rate of dissipation is reached, where very little difference is seen between the curves. At each time, the greatest rate of energy dissipation is found low down, near the photosphere. This is not surprising when we consider the equation describing $Q$ (Equation 6). From this equation we see that $Q_{\text {frc }}$ and $Q_{\text {hd }}$ are both proportional to magnetic field strength, $|\mathbf{B}|, Q_{\text {frc }}$ is also proportional to velocity, $|\mathbf{v}|$, and $Q_{\text {hd }}$ to gradients in $\alpha$. Figure 6(f) shows a plot of the total absolute flux through surfaces of constant $z$, as a function of height. This is shown at $t=168 \mathrm{hr}$, but similar curves are seen throughout the simulation. This is computed as:

$$
\phi(z)=\int_{y_{\min }}^{y_{\max }} \int_{x_{\min }}^{x_{\max }}\left|B_{z}(x, y, z)\right| d x d y .
$$

The flux is greatest low down, near the magnetic sources, then drops of rapidly with increasing height. This indicates that most connections between magnetic features close low down. From Figure 6(e), the rate of energy dissipation also rapidly drops with increasing height, having decreased by more than an order of magnitude by $z=2 \mathrm{Mm}$. Therefore, the energy dissipation term $Q$ has its largest effect close to the photosphere. In contrast to energy dissipated (which from Equation 6 is always $>0$ ), localised values of free magnetic energy density can be either positive or negative. In Figure 3(e) and (f) black patches can be seen low down, indicating locations of negative free energy density, most likely occurring due to the cancellation of magnetic features on the photosphere removing energy. Higher up, the free energy density tends to be only positive (white patches). The combined effect of positive and negative regions low down results in the peak in total free energy density as a function of height occurring higher up, just below $1 \mathrm{Mm}$ (Figure 3(b)), where the free energy density becomes solely positive. The movie, magnet48b_q_ht.mpg, shows the rate of energy dissipation as a function of height for the first $8.3 \mathrm{hr}$ of the $3 \mathrm{G}$ simulation. At the start of the movie, at greater heights, the rate of energy dissipation gradually increases until the curve becomes more or less steady. One can see occasional kinks in the curve at low $z$, which then propagate upward.

Figures 7(a) and (b) show images of $Q$ integrated in $z$, in the $x-y$ plane, from the $3 \mathrm{G}$ simulation. This is computed with units of ergs $\mathrm{cm}^{-2} \mathrm{~s}^{-1}$ as follows:

$$
E_{\mathrm{q}}(x, y)=\int_{z_{\min }}^{z_{\max }} Q(x, y, z) d z .
$$


(a)

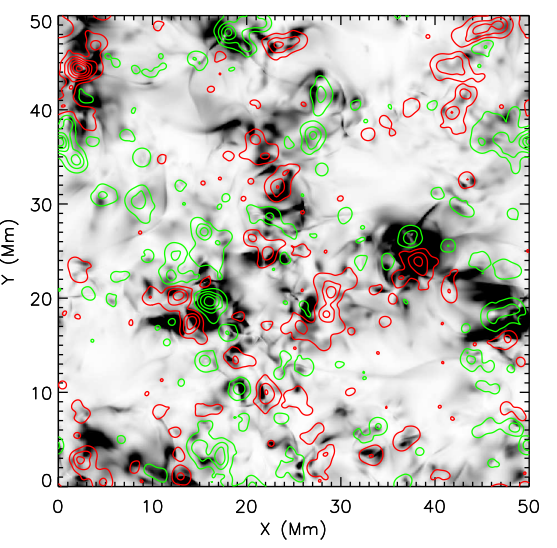

(c)

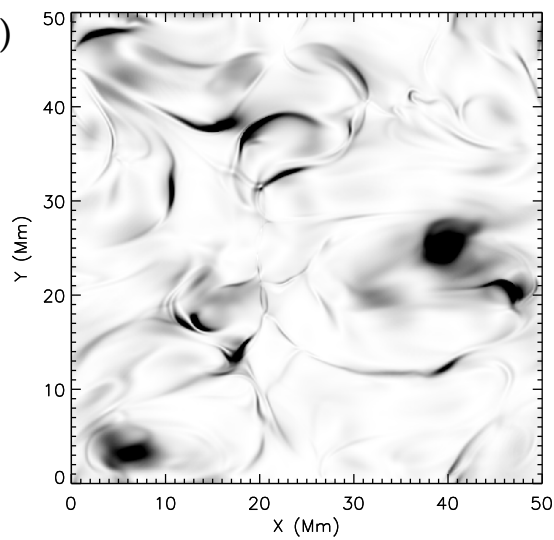

(e)

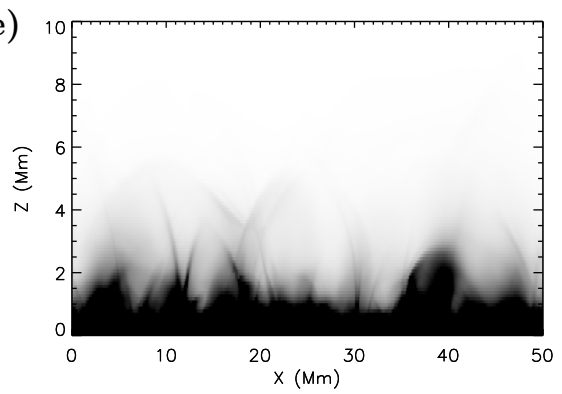

(b)

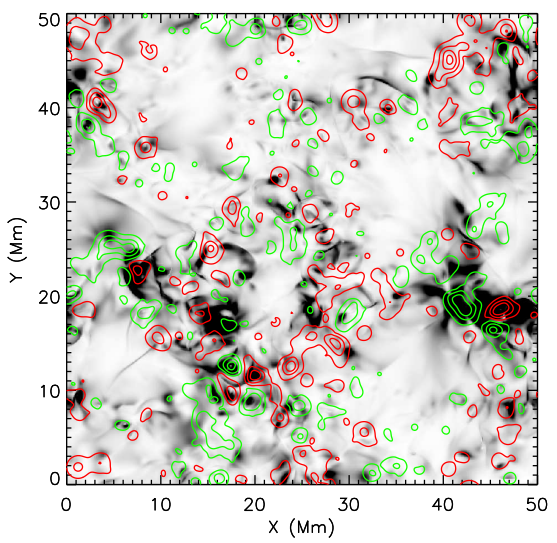

(d)

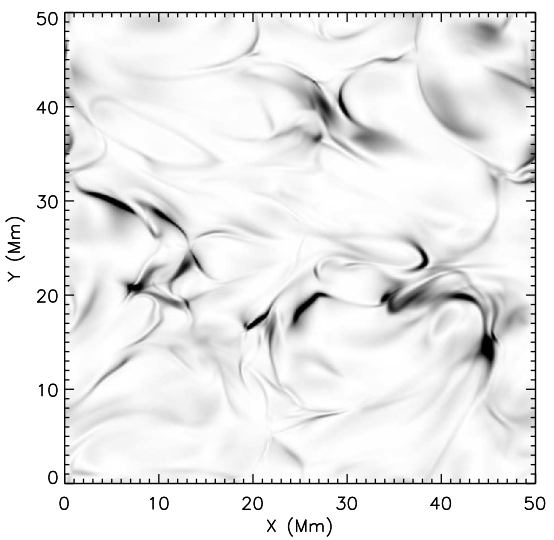

(f)

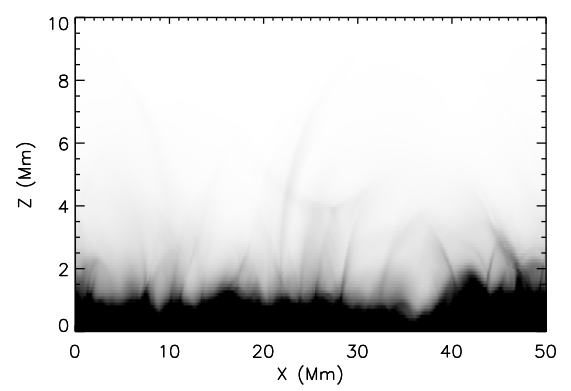

Figure 7. All images are for the $3 \mathrm{G}$ simulation. Darker regions correspond to higher values. (a) and (b) Rate of energy dissipation, $Q$, integrated in $z$. The images are shown in the $x-y$ plane, saturated at $1.5 \times 10^{5} \mathrm{ergs} \mathrm{cm}^{-2} \mathrm{~s}^{-1}$. Positive (red) and negative (green) contours of $B_{z}$ at $z=0 \mathrm{Mm}$ are over-plotted at levels of $\pm[7,13,27,53,106] \mathrm{G}$. (c) and (d) Rate of energy dissipation, $Q$, saturated at $810 \mathrm{ergs} \mathrm{cm} \mathrm{cs}^{-2} \mathrm{~s}^{-1}$, shown in the $x-y$ plane at $z=3 \mathrm{Mm}$. (e) and (f) Images of $Q$ in the $x-z$ plane integrated in $y$, saturated at $1.5 \times 10^{5} \mathrm{ergs} \mathrm{cm}^{-2} \mathrm{~s}^{-1}$. Images in the left-hand column are shown at $t=128 \mathrm{hr}$ and in the right-hand column at $t=168 \mathrm{hr}$. 
As in Figure 5, the colour table has been reversed, so that darker regions correspond to higher values of $E_{\mathrm{q}}(x, y)$. The images are shown at (a) $t=128$ $\mathrm{hr}$ and (b) $t=168 \mathrm{hr}$, and are saturated at a level of $1.5 \times 10^{5} \mathrm{ergs} \mathrm{cm}^{-2}$ $\mathrm{s}^{-1}$. As in Figures $3(\mathrm{c})$ and (d), contours of $B_{z}$ at $z=0 \mathrm{Mm}$ are over-plotted. One can see that $Q$ is more localised than the free magnetic energy. Patches of $Q$ tend to lie around the magnetic network, but not necessarily at the same locations as free magnetic energy seen in Figures 3(c) and (d). In addition, we do not see far-reaching bands of $Q$ across the supergranules as we did with the free energy. In Paper II, it was determined that $Q$ is mainly located at sites of changing magnetic connectivity and low down, near the magnetic elements. Therefore, it is unsurprising that in these simulations, $Q$ is seen mainly at the magnetic network, as this is where large numbers of magnetic elements lie, and the magnetic connectivity is constantly changing due to the continual interaction of these elements with one another.

A movie showing $Q$ in the $x-y$ plane for the $3 \mathrm{G}$ simulation is available (magnet48b_q_xy_bz.mpg). It shows $Q$ integrated in $z$, and saturated at $1.5 \times 10^{5}$ ergs $\mathrm{cm}^{-2} \mathrm{~s}^{-1}$, with contours of $B_{z}$ at $z=0 \mathrm{Mm}$ over-plotted. The movie shows that the locations and evolution of $Q$ are very different from those of the positive free magnetic energy density (magnet48b_free_xy_bz.mpg). Indeed, while many of the patches of positive free magnetic energy density were seen to be long-lived, regions of $Q$ are seen to be very short-lived, occurring in rapidly evolving 'bursts'. Within the free energy density movie, patches of positive free energy density are often seen stretched across the supergranular cells, whereas $Q$ tends to be much more localised, occurring predominantly within the magnetic network where large magnetic elements lie. Several large bursts of $Q$ can be seen throughout the movie, in regions where many magnetic elements are emerging and interacting with one another. For readers unable to view the movie, six still images from the movie spaced $1 \mathrm{hr}$ apart are included in Appendix A (Figure 10). It can be seen that the spatial distribution of $Q$ integrated in the LOS changes significantly from one hour to the next. Note that the evolution of $Q$ that we see in these movies is dominated by $Q$ low down, since the rate of energy dissipation rapidly decreases with increasing $z$ (Figures 6(e) and (f)). We now consider the spatial location of $Q$ as a function of height above the photosphere.

Figures $7(\mathrm{c})$ and $(\mathrm{d})$ show $x-y$ plane images of $Q$ at $z=3 \mathrm{Mm}$ in the $3 \mathrm{G}$ simulation, at $t=128 \mathrm{hr}$ and $t=168 \mathrm{hr}$ respectively. $Q$ at height $z=3 \mathrm{Mm}$ is given by $Q(x, y, 3) L_{z}$. As in Figures 7(a) and (b), many patches of $Q$ can be seen located above the boundaries between supergranules. However, in contrast to Figures 7(a) and (b), within Figures 7(c) and (d) one can also see much more fine-scale structure to $Q$ when it is not integrated along the line of sight. Also in contrast to Figures 7 (a) and (b), where $Q$ integrated over $z$ is predominantly located at the magnetic network, here we see long strands of $Q$ which lie across the supergranules at $z=3 \mathrm{Mm}$. These are localised regions of energy dissipation that are found mainly at sites of changing magnetic connectivity. A movie of $Q$ above the photosphere is available (magnet48b_q_xy_3_10.mpg) showing $Q$ in the $x-y$ plane, integrated between $z=3 \mathrm{Mm}$ and $z=10 \mathrm{Mm}$ :

$$
E_{\mathrm{q}, 3-10}(x, y)=\int_{z=3 \mathrm{Mm}}^{z=10 \mathrm{Mm}} Q(x, y, z) d z,
$$


in units of ergs cm $\mathrm{cm}^{-2} \mathrm{~s}^{-1}$. The movie is saturated at $3 \times 10^{3} \mathrm{ergs} \mathrm{cm}^{-2} \mathrm{~s}^{-1}$. At this height, the rate of energy dissipation is much lower than at the photosphere, as the coronal field is evolving more slowly and is less non-potential. The result of this is that $Q$ is also less rapidly evolving than it is lower down. In addition, between these heights, $Q$ is seen to occur anywhere within the $x-y$ plane and not just above the magnetic network. We also see more fine-scale, further reaching structures. Six images of $Q$ at $z=3 \mathrm{Mm}$ spaced $1 \mathrm{hr}$ apart are also included in Appendix A (Figure 11), to illustrate the time-scale of evolution at this height.

Figures $7(\mathrm{e})$ and (f) show $x-z$ plane images of $Q$ integrated in $y$, saturated at $1.5 \times 10^{5} \mathrm{ergs} \mathrm{cm}^{-2} \mathrm{~s}^{-1}$, at $t=128 \mathrm{hr}$ and $t=168 \mathrm{hr}$, respectively. In agreement with Figures 6(e) and (f), the energy dissipation is seen to be greatest low down. Finer-scale structure can then be seen between $z=2.5 \mathrm{Mm}$ and $z=5 \mathrm{Mm}$. Two movies of $Q$ in the $x-z$ plane for the $3 \mathrm{G}$ simulation are included with this paper. Similar evolution is seen in the $y-z$ plane and for other strengths of overlying field. The first movie, magnet48b_q_xz.mpg, shows $Q$ integrated in $y$ and saturated at the same level as in Figures $7(\mathrm{e})$ and (f). The second movie, magnet48b_q_xz_log.mpg, shows the logarithm of the first so that the energy dissipation can be seen for a wider range of values. From this it can be seen that fine-scale structures also exist higher up in the corona, where the energy dissipation is much weaker. In both movies, occasional 'bursts' can be seen, where a feature will drift upwards and disappear (or rather, become too faint to be shown at the current level of saturation). From these movies, we find that the energy dissipated and summed along the line of sight provides a fine-scale dynamic structure that is in qualitative agreement with what is observed on the Sun low down.

\section{Discussion and Conclusions}

The aim of this paper was to carry out a preliminary analysis of a set of smallscale, non-linear force-free field simulations. The simulations were driven by synthetic magnetograms produced by the model described in Paper I. Four simulations were run, each driven by the same lower boundary data, three with different strengths of overlying field: $1 \mathrm{G}, 3 \mathrm{G}$ and $10 \mathrm{G}$, and one with no overlying field. The lower boundary data consisted of a $48 \mathrm{hr}$ series of synthetic magnetograms of area $50 \times 50 \mathrm{Mm}^{2}$ and of cadence $1 \mathrm{~min}$. The initial coronal magnetic field for each simulation was potential. This field was then evolved through a series of quasi-static, non-linear force-free equilibria, via a magnetofrictional relaxation technique, in response to photospheric boundary motions. The continuous nature of this coronal evolution technique means that current systems are maintained within the corona from one step to the next and the evolution is smooth. This allows for the build-up and storage of free magnetic energy - one of the quantities studied within this paper. The presence of free magnetic energy within our model shows a significant departure from previous models of the magnetic carpet coronal field, which use potential fields. The other quantities considered were the energy dissipated and the square of the electric current density, $j^{2}$. 
Initially, for each simulation, both the free magnetic energy and energy dissipation rate rapidly increase, before levelling off and oscillating about a mean value. The mean free magnetic energy per unit area for each simulation is $6.5-11.7 \times 10^{7} \mathrm{ergs} \mathrm{cm}^{-2}$, whilst the mean energy dissipation rate is $3.1-4.2 \times 10^{4}$

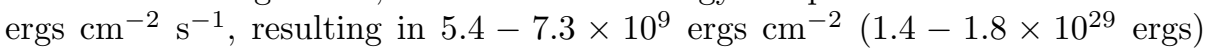
being cumulatively dissipated over each $48 \mathrm{hr}$ simulation. For both the free and dissipated energies, a stronger overlying field results in higher values, although the effect is more significant for the free energy. It is also clear that the evolution of both the free and dissipated energies is highly dependent upon the evolution of the photospheric magnetic field.

While there are similarities between the evolution of the two types of energy integrated over the volume, they are seen to be less alike when we consider their location within each simulation. The bulk of the free magnetic energy is located above the photosphere, between $z=0.5-0.8 \mathrm{Mm}$. This is stored along closed connections between magnetic elements. Regions of positive free energy density can be seen both in the magnetic network and across supergranular cells; such regions may also be long-lived. In contrast, the largest amount of energy dissipation is found low down, near the magnetic sources, and values decrease rapidly with increasing height. Regions of increased energy dissipation are seen predominantly in the magnetic network, although weaker, fine-scale strands are also seen above the photosphere at sites of changing magnetic connectivity. Also unlike the free magnetic energy density, the large regions of energy dissipation seen in the $x-y$ plane are much more rapidly evolving (compare movies magnet48b_free_xy_bz.mpg and magnet48b_q_xy_bz.mpg).

The amount of free magnetic energy built up and stored in each simulation $\left(1.1-2.1 \times 10^{27} \mathrm{ergs}\right)$ is sufficient to account for such small-scale transient phenomena as nanoflares ( $\sim 10^{24}$ ergs, Parker, 1988$)$ and X-ray bright points $\left(10^{22}-10^{24}\right.$ ergs s$^{-1}$, Habbal and Withbroe, 1981; Longcope, 1998). The energy dissipation rate is not high enough to be able to explain the heating of the quiet corona, providing a contribution of around $31-42 \%$ to the required $10^{5}$ ergs $\mathrm{cm}^{-2} \mathrm{~s}^{-1}$ of Withbroe and Noyes (1977) or $6.3-8.6 \%$ to the required $4.9 \times 10^{5}$ $\operatorname{ergs} \mathrm{cm}^{-2} \mathrm{~s}^{-1}$ of Habbal and Grace (1991). In fact, the discrepancy may be even larger since Habbal and Grace (1991) did not include the energy losses due to thermal conduction. However, the location and structure of regions of energy dissipation within our model are at least in qualitative agreement with what is observed on small scales on the Sun. The lower rate of energy dissipation may in part be due to the simplified magnetofrictional model that has been applied, but also due to an insufficient rate of energy being injected in the first place. An order of magnitude estimate of the Poynting flux injected showed that this was within a factor or $2-3$ of the mean energy dissipated in each simulation. This indicates that increasing the magnitude of the horizontal velocities or the horizontal or vertical magnetic field components at the photosphere, hence increasing the Poynting flux, could result in higher energy dissipation. Another reason for our Poynting flux (and hence energy dissipated) being lower than observed values is that in the present model, our magnetic features are treated as 'rigid' bodies. If the magnetic field of an actual feature were instead contained within many intense kilogauss flux tubes, the movement of features would be more fluid (as is 
Table 3. Summary of results for free magnetic energy; energy dissipated, $Q$, and current density, $j^{2}$.

\begin{tabular}{|c|c|c|c|}
\hline Quantity & Location & $\begin{array}{l}\text { Variation } \\
\text { in height }\end{array}$ & $\begin{array}{l}\text { Effect of } \mathrm{O} / \mathrm{L} \\
\text { field strength }\end{array}$ \\
\hline $\begin{array}{c}\text { Free } \\
\text { Energy }\end{array}$ & $\begin{array}{l}\text { Magnetic network and } \\
\text { across s/g cells. }\end{array}$ & $\begin{array}{c}\text { Mostly stored } z=0.5-0.8 \mathrm{Mm} \\
\text { then rapid decrease } \\
\text { with increasing } z .\end{array}$ & $\begin{array}{c}\text { Significant } \\
\text { increase in } \\
\text { total with } \\
\text { increasing } B_{0}\end{array}$ \\
\hline$Q$ & $\begin{array}{l}\text { Magnetic network and } \\
\text { sites of changing } \\
\text { magnetic connectivity. }\end{array}$ & $\begin{array}{l}\text { Rapid decrease with } \\
\quad \text { increasing } z\end{array}$ & $\begin{array}{l}\text { Slight } \\
\text { increase in } \\
\text { total with } \\
\text { increasing } B_{0}\end{array}$ \\
\hline$j^{2}$ & $\begin{array}{c}\text { Magnetic network and } \\
\text { non-potential regions. } \\
\text { Follows field lines. }\end{array}$ & - & - \\
\hline
\end{tabular}

observed) as the intense flux tubes have high local velocities. This would lead to a greater injection of Poynting flux. It should also be noted that our model does not include a chromosphere. Habbal and Grace (1991) determined chromospheric radiative losses to be $3.2 \times 10^{5} \mathrm{ergs}^{-2} \mathrm{sm}^{-1}$, while the value determined by Withbroe and Noyes (1977) is an order of magnitude higher at $4 \times 10^{6} \mathrm{ergs} \mathrm{cm}^{-2}$ $\mathrm{s}^{-1}$. A more complex model that included a chromosphere with the effects of, for example, Alfvén waves and turbulence would likely result in a much higher rate of energy dissipation (e.g. van Ballegooijen et al. (2011)).

Locations of increased $j^{2}$ are found to be co-located with regions of positive free magnetic energy density, as both are dependent on the magnetic field being non-potential. Visually, $j^{2}$ is seen to follow the shape of the magnetic field where $\alpha$ is non-zero. Table 3 gives a summary of results for each of the quantities studied in this paper, indicating their locations and the effect of increasing the strength of the overlying field.

There are several avenues for future work using the non-linear force-free coronal modelling technique described here. The simulations presented in this paper will be studied in more detail. For example, a more in depth study of the connectivity of the magnetic field will be conducted, similar to the study of Close et al. (2003), who analysed potential coronal fields. It would also be of interest to investigate in detail the effect of each of the photospheric flux evolution processes on the evolution of the coronal field and on each of the energy quantities discussed in this paper. We already know the flux, location and time of occurrence of each of the processes within the synthetic magnetogram series. Further 2D simulations will also be run to produce synthetic magnetograms that include an evolving supergranular flow profile and magnetic features on smaller scales. The added complexity of evolving photospheric flows and smaller 
magnetic features will likely result in a larger build up of free magnetic energy and greater energy dissipation, when the coronal field evolution is simulated.

The rate of energy dissipation per unit area produced by our present simulations was found to be too low to explain quiet Sun radiative losses. This is unsurprising, however, as many simplifications have been made for our coronal model and it is not a true physical energy dissipation - we do not have currently have an energy equation. For example, the present simulations do not include the effects of small-scale magnetic braiding and MHD waves, which are believed to be important for heating the chromosphere and corona in active regions (van Ballegooijen et al., 2011; Asgari-Targhi and van Ballegooijen, 2012). In future we intend to extend the present magnetofrictional model to contain plasma by including the pressure and density terms in the equations of magnetohydrodynamics. We will then be able to follow the corresponding plasma processes associated with energy dissipation and give an estimate of the resultant plasma heating.

The magnetofrictional technique will also be applied to real magnetogram data, such as from Hinode/SOT or SDO/HMI. A study will be carried out to compare regions of interest within a simulated non-linear force-free coronal field driven by observed magnetograms to events occurring in corresponding coronal images (e.g. using various wavelengths of $\mathrm{SDO} / \mathrm{AIA}$ ). What is clear is that within this single, relatively 'simple' simulation, a wide range of processes and dynamics is occurring. A careful and detailed analysis of these and other non-linear forcefree field simulations of the magnetic carpet coronal field will be carried out in future.

Acknowledgements KAM and DHM gratefully acknowledge the support of the Leverhulme Trust and the STFC. DHM would like to thank the Royal Society for their support through the Research Grant Scheme. DHM and CEP acknowledge support from the EU under FP7.

\section{Appendix}

\section{A. Images From Movies}

This appendix contains four figures, each with six images spaced $1 \mathrm{hr}$ apart from $t=144 \mathrm{hr}$ to $t=149 \mathrm{hr}$, taken from some of the $x-y$ plane movies included with this paper.

\section{References}

Asgari-Targhi, M., van Ballegooijen, A.A.: 2012, Astrophys. J. 746, 81

Aschwanden, M.J., De Pontieu, B., Schrijver, C.J., Title, A.: 2002, Solar Phys. 206, 99.

Berger, M.A., Asgari-Targhi, M.: 2009, Astrophys. J. 705, 347.

Boozer, A.H.: 1986, J. Plasma Phys. 35, 133.

Close, R.M., Parnell, C.E., Mackay, D.H., Priest, E.R.: 2003, Solar Phys. 212, 251.

Close, R.M., Parnell, C.E., Longcope, D.W., Priest, E.R.: 2004, Astrophys. J. Lett. 612, L81.

Cranmer, S.R., van Ballegooijen, A.A.: 2010, Astrophys. J. 720, 824

DeForest, C E., Hagenaar, H.J., Lamb, D.A., Parnell, C.E., Welsch, B.T.: 2007, Astrophys. J. 666, 576. 
(a)

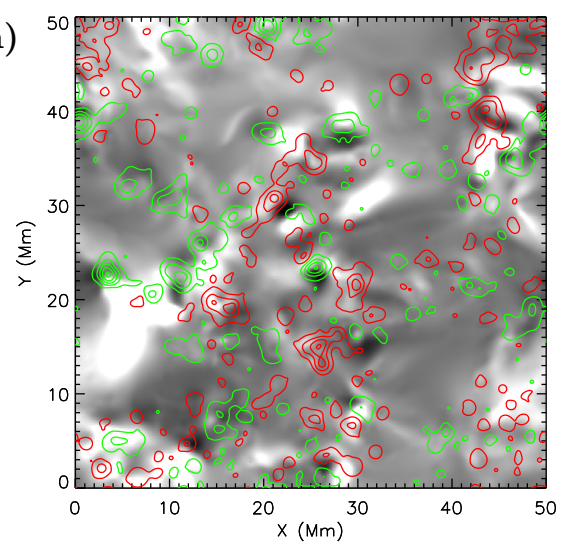

(c)

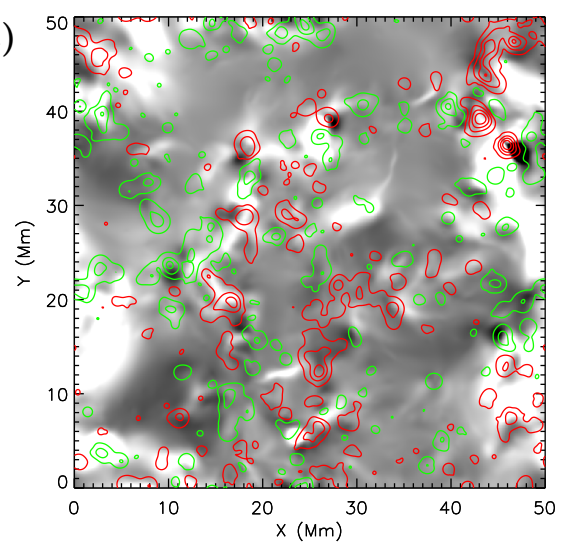

(e)

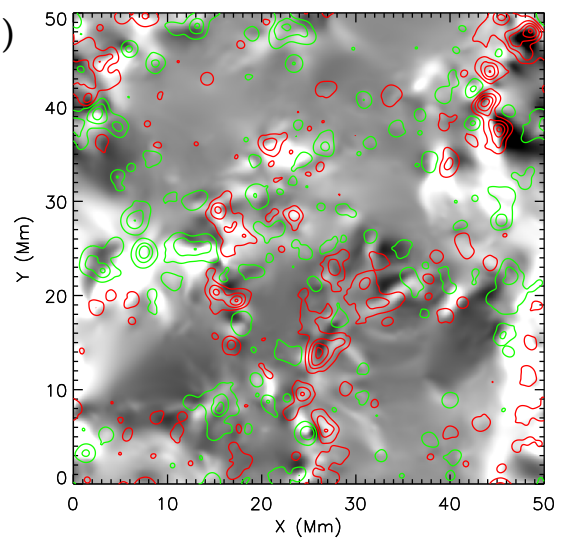

(b)

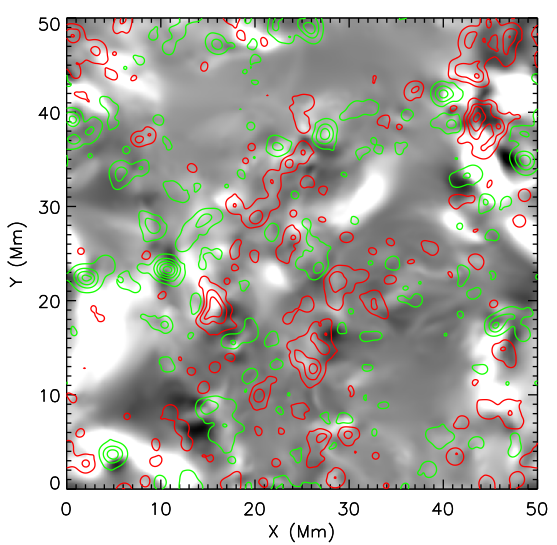

(d)

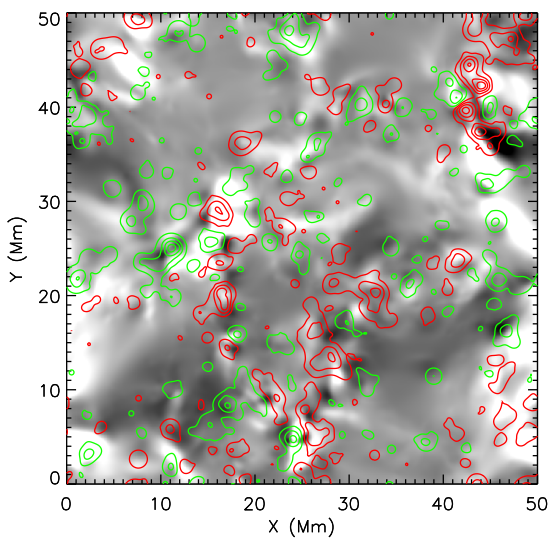

(f)

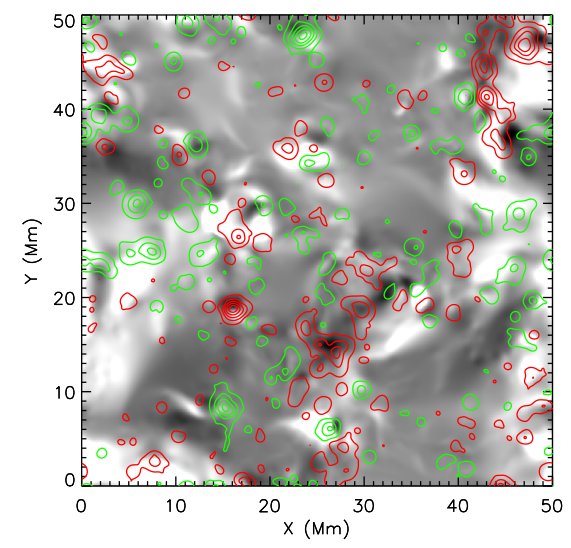

Figure 8. Free magnetic energy density integrated in the line-of-sight, for the $3 \mathrm{G}$ overlying field simulation. The images are shown in the $x-y$ plane saturated at $\pm 1.9 \times 10^{22}$ ergs. Contours of $B_{z}$ at $z=0 \mathrm{Mm}$ are over-plotted where red contours represent positive magnetic field and green contours represent negative, at levels of $\pm[7,13,27,53,106] \mathrm{G}$. The images are shown at (a) $t=144 \mathrm{hr}$, (b) $t=145 \mathrm{hr}$, (c) $t=146 \mathrm{hr}$, (d) $t=147 \mathrm{hr}$, (e) $t=148 \mathrm{hr}$ and (f) $t=149 \mathrm{hr}$. 
(a)

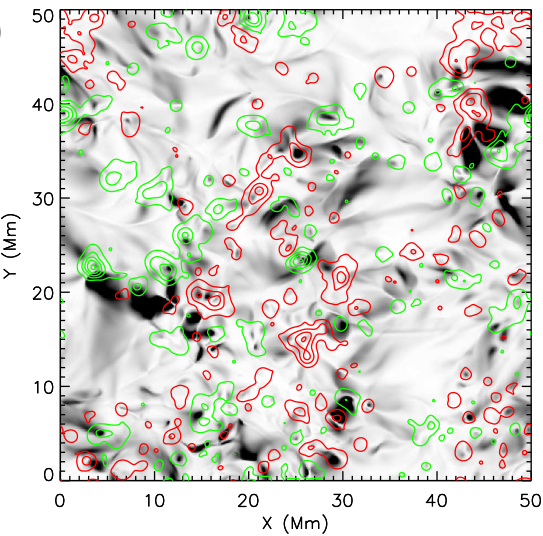

(c)

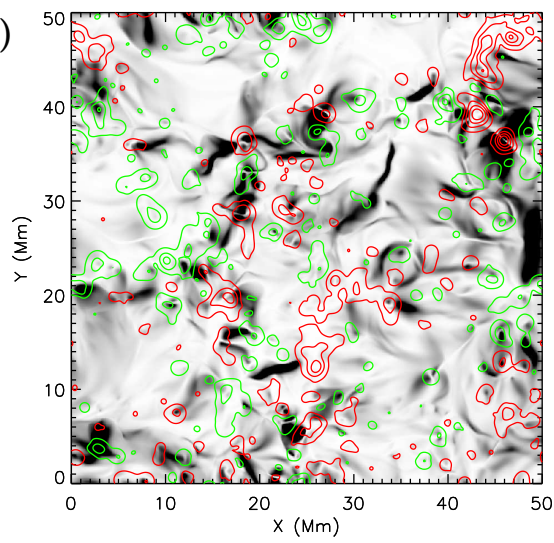

(e)

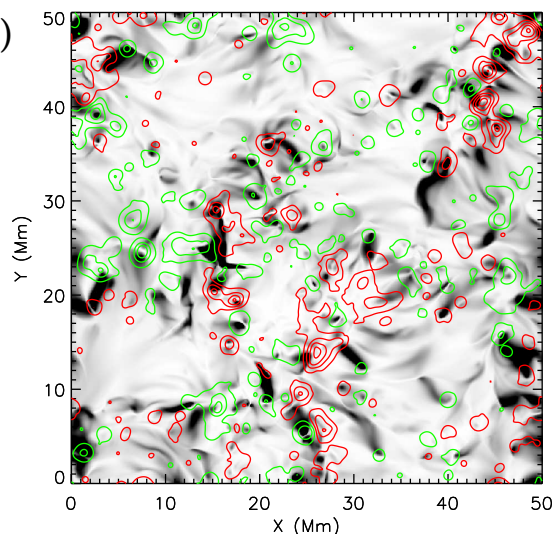

(b)

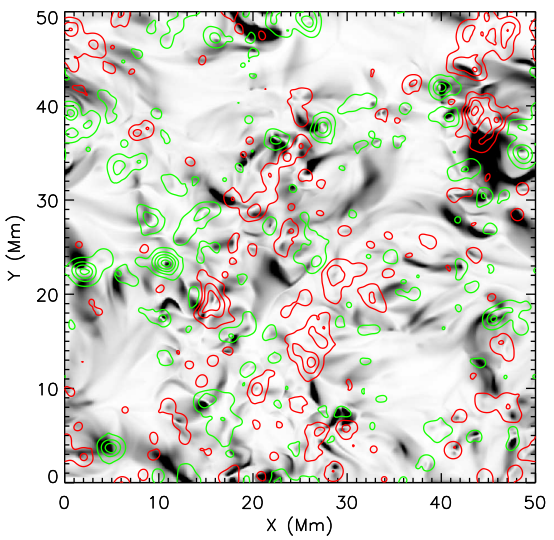

(d)

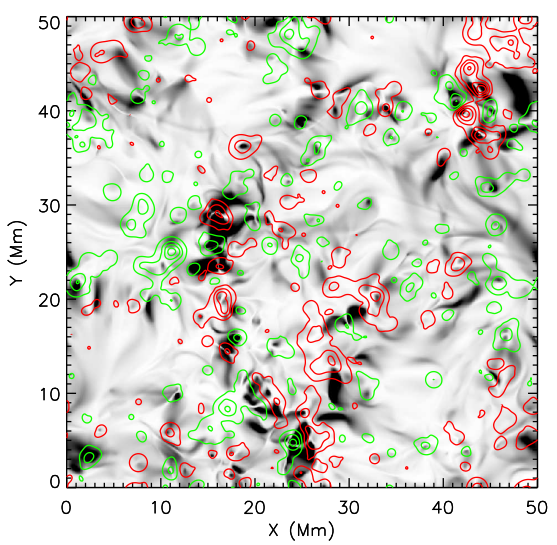

(f)

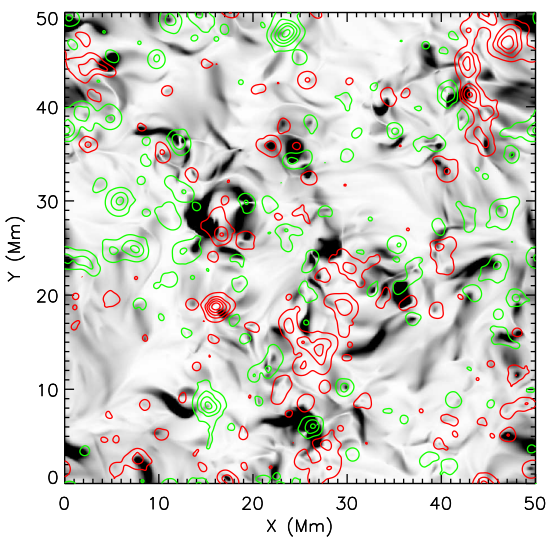

Figure 9. Normalised $j^{2}$ integrated in $z$, shown in the $x-y$ plane, for the $3 \mathrm{G}$ overlying field simulation. Darker regions correspond to higher values. Contours of $B_{z}$ at $z=0 \mathrm{Mm}$ are over-plotted where red contours represent positive magnetic field and green contours represent negative, at levels of $\pm[7,13,27,53,106]$ G. The images are shown at (a) $t=144 \mathrm{hr}$, (b) $t=145$ $\mathrm{hr}$, (c) $t=146 \mathrm{hr}$, (d) $t=147 \mathrm{hr}$, (e) $t=148 \mathrm{hr}$ and (f) $t=149 \mathrm{hr}$. 
(a)

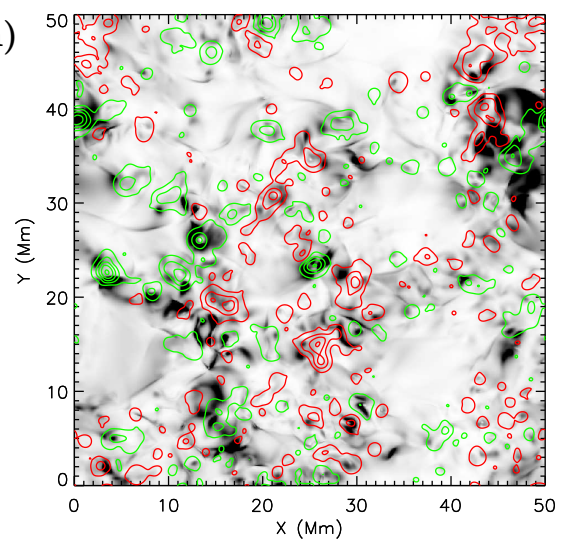

(c)

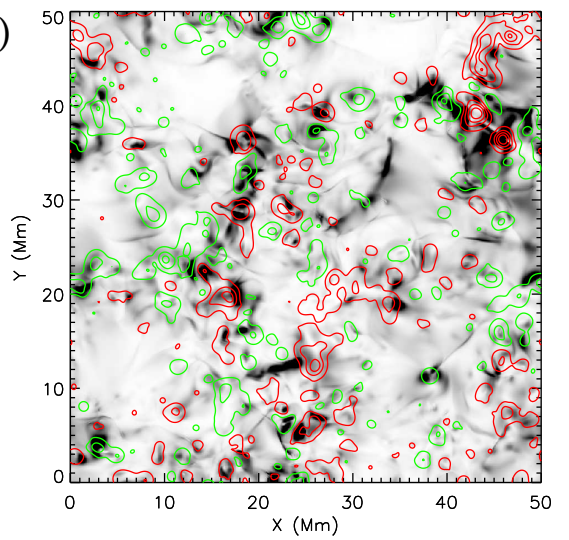

(e)

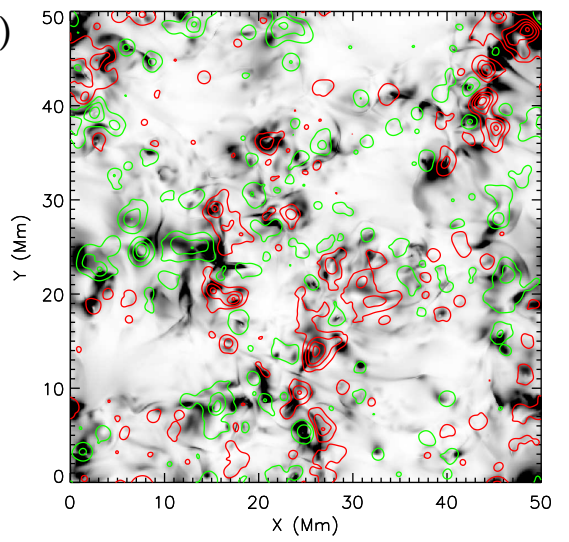

(b)

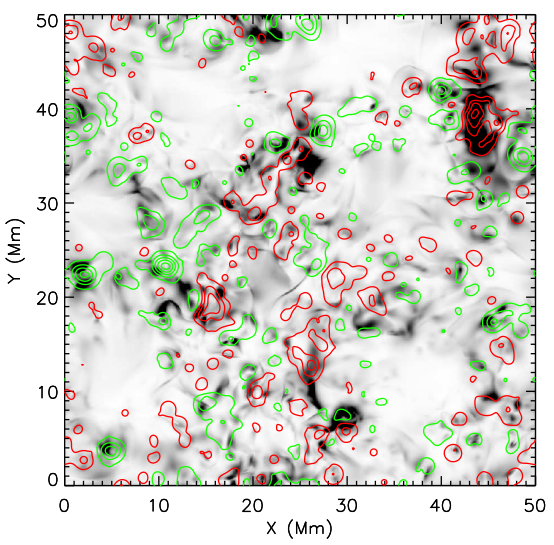

(d)

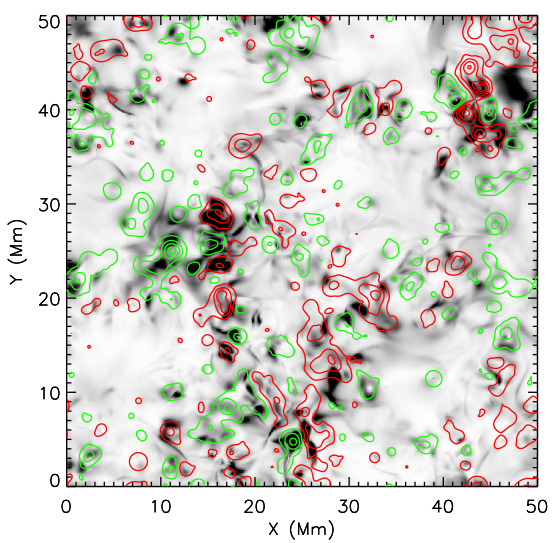

(f)

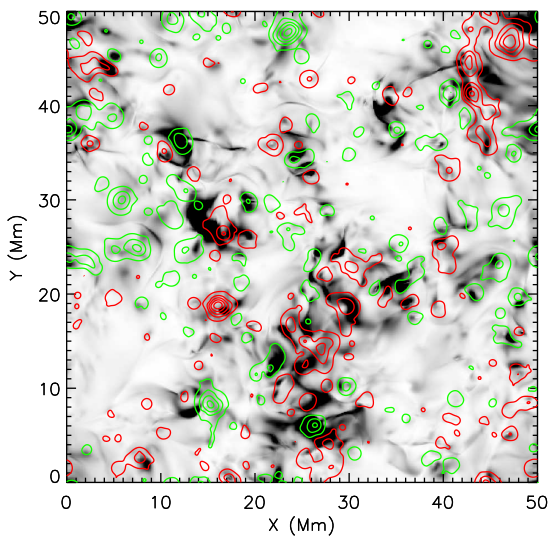

Figure 10. Rate of energy dissipation, $Q$, integrated in the line-of-sight, for the $3 \mathrm{G}$ overlying field simulation. Darker regions correspond to higher values. The images are shown in the $x-y$ plane saturated at $1.5 \times 10^{5} \mathrm{ergs} \mathrm{cm}^{-2} \mathrm{~s}^{-1}$. Contours of $B_{z}$ at $z=0 \mathrm{Mm}$ are over-plotted where red contours represent positive magnetic field and green contours represent negative, at levels of $\pm[7,13,27,53,106] \mathrm{G}$. The images are shown at (a) $t=144 \mathrm{hr}$, (b) $t=145 \mathrm{hr}$, (c) $t=146 \mathrm{hr}$, (d) $t=147 \mathrm{hr}$, (e) $t=148 \mathrm{hr}$ and (f) $t=149 \mathrm{hr}$. 
(a)

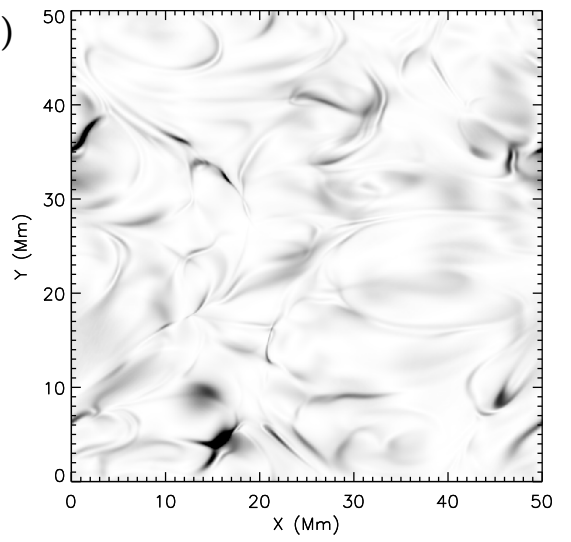

(c)

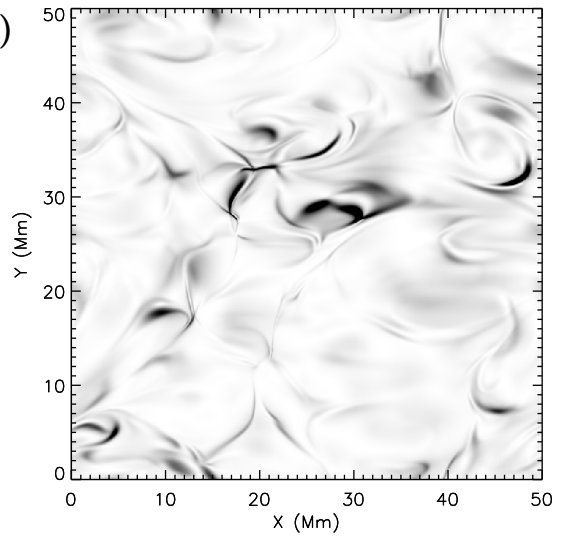

(e)

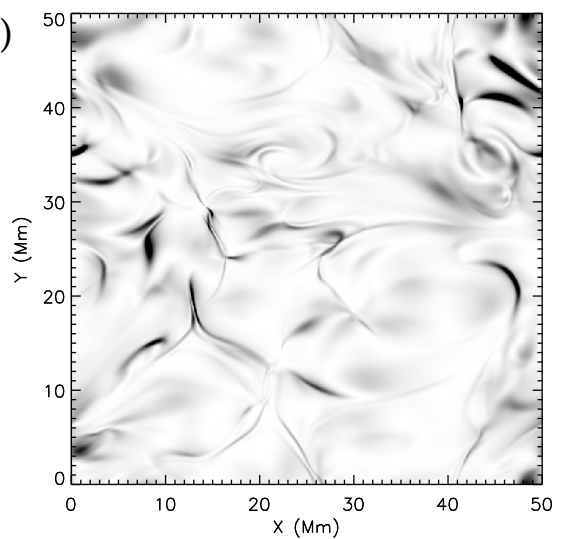

(b)

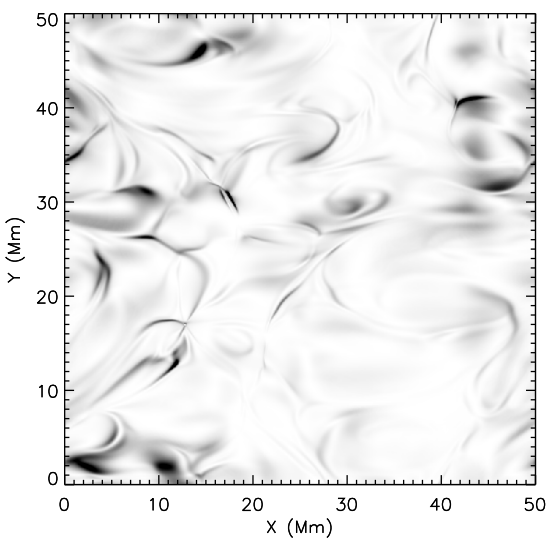

(d)

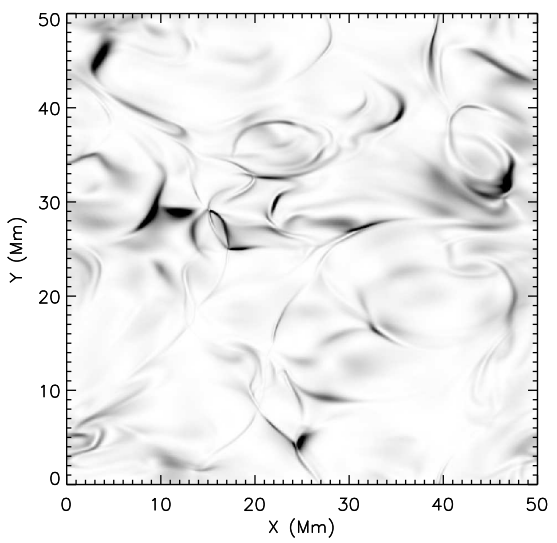

(f)

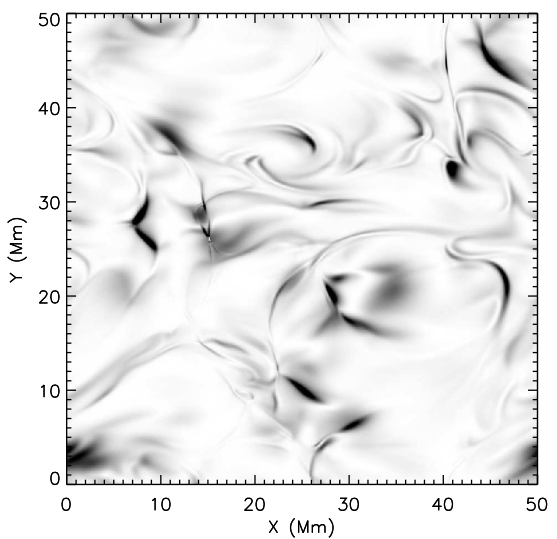

Figure 11. Rate of energy dissipation, $Q$, for the $3 \mathrm{G}$ overlying field simulation. The images are shown in the $x-y$ plane at $z=3 \mathrm{Mm}$, saturated at $810 \mathrm{ergs} \mathrm{cm}^{-2} \mathrm{~s}^{-1}$. Darker regions correspond to higher values. They are shown at (a) $t=144 \mathrm{hr}$, (b) $t=145 \mathrm{hr}$, (c) $t=146 \mathrm{hr}$, (d) $t=147 \mathrm{hr}$, (e) $t=148 \mathrm{hr}$ and (f) $t=149 \mathrm{hr}$. 
De Moortel, I., Pascoe, D.J.: 2009, Astrophys. J. Lett. 699, L72.

de Wijn, A.G., Lites, B.W., Berger, T.E., Frank, Z.A., Tarbell, T.D., Ishikawa, R.: 2008, Astrophys. J. 684, 1469.

Galsgaard, K., Nordlund, Å.: 1996, Astrophys. Lett. Comm. 34, 175.

Habbal, S.R., Grace, E.: 1991, Astrophys. J. 382, 667.

Habbal, S.R., Withbroe, G.L.: 1981, Solar Phys. 69, 77.

Hagenaar, H.J., DeRosa, M.L., Schrijver, C.J.: 2008, Astrophys. J. 678, 541.

Harvey, K.L., Martin, S.F.: 1973, Solar Phys. 32, 389.

Harvey-Angle, K.L.: 1993, Ph.D. thesis, Utrecht University.

Haynes, A.L., Parnell, C.E., Galsgaard, K., Priest, E.R.: 2007, Roy. Soc. Lond. Proc. Ser. A 463, 1097.

Leighton, R.B., Noyes, R.W., Simon, G.W.: 1962, Astrophys. J. 135, 474.

Livingston, W.C., Harvey, J.: 1975, Bull. Amer. Astron. Soc. 7, 346.

Longcope, D.W.: 1998, Astrophys. J. 507, 433.

Longcope, D.W., Parnell, C.E.: 2009, Solar Phys. 254, 51.

Mackay, D.H., Green, L.M., van Ballegooijen, A.A. : 2011, Astrophys. J. 729, 97.

Mackay, D.H., van Ballegooijen, A.A.: 2006, Astrophys. J. 641, 577.

Mackay, D.H., van Ballegooijen, A.A.: 2009, Solar Phys. 260, 321.

Martin, S.F.: 1984, In: Keil, S. L. (ed.) Small Scale Dynamical Processes in Quiet Stellar Atmospheres, National Solar Observatory, Sunspot, 30.

Martin, S.F.: 1988, Solar Phys. 117, 243.

Meyer, K.A., Mackay, D.H., van Ballegooijen, A.A., Parnell, C.E.: 2011, Solar Phys. 272, 29.

Meyer, K.A., Mackay, D.H., van Ballegooijen, A.A. : 2012, Solar Phys. 278, 149.

Nakariakov, V.M., Ofman, L.: 2001, Astron. Astrophys. 372, L53.

Parker, E.N.: 1988, Astrophys. J. 330, 474.

Parnell, C.E., Galsgaard, K.: 2004, Astron. Astrophys. 428, 595.

Parnell, C.E., De Moortel, I.: 2012, Phil. Trans. R. Soc. A 370, 3217.

Priest, E.R., Heyvaerts, J.F., Title, A.M.: 2002, Astrophys. J. 576, 533.

Pontin, D.I., Wilmot-Smith, A.L., Hornig, G., Galsgaard, K.: 2011, Astron. Astrophys. 525, A57.

Rappazzo, A.F., Velli, M., Einaudi, G., Dahlburg, R.B : 2008, Astrophys. J. 677, 1348.

Régnier, S., Parnell, C.E., Haynes, A.L.: 2008, Astron. Astrophys. 484, L47.

Rieutord, M., Rincon, F.: 2010, Living Rev. Solar Phys. 7(2).

Schrijver, C.J.: 2010, Astrophys. J. 710, 1480.

Schrijver, C.J., Hagenaar, H.J., Title, A.M.: 1997, Astrophys. J. 475, 328.

Schrijver, C.J., Title, A.M.: 2002, Astrophys. J. 207, 223.

Simon, G.W., Leighton, R.B.: 1964, Astrophys. J. 140, 1120.

Thornton, L.M., Parnell, C.E.: 2011, Solar Phys. 269, 13.

van Ballegooijen, A.A., Cranmer, S.R.: 2008, Astrophys. J. 682, 644.

van Ballegooijen, A.A., Priest, E.R., Mackay, D.H.: 2000, Astrophys. J. 539, 983.

van Ballegooijen, A.A., Asgari-Targhi, M., Cranmer, S.R., DeLuca, E.E.: 2011, Astrophys. J. 736, 3 .

van Ballegooijen, A.A., Nisenson, P., Noyes, R.W., Löfdahl, M.G., Stein, R.F., Nordlund, A., Krishnakumar, V.: 1998, Astrophys. J. 509, 435.

Van Doorsselaere, T., Nakariakov, V.M., Young, P.R., Verwichte, E.: 2008, Astron. Astrophys. 487, L17.

Verwichte, E., Nakariakov, V.M., Ofman, L., DeLuca, E.E.: 2004 Solar Phys. 223, 77.

Wang, H., Zirin, H.: 1989 Solar Phys. 120, 1.

Wang, T.J., Inees, D.E., Qiu, J.: 2007, Astrophys. J. 656, 598.

Wang, H., Tang, F., Zirin, H., Wang, J.: 1996 Solar Phys. 165, 223.

West, M.J., Zhukov, A.N., Dolla, L., Rodriguez, L.: 2011, Astrophys. J. 730, 122.

Wilmot-Smith, A.L., Hornig, G., Priest, E.R.: 2009 Geophys. Astrophys. Fluid Dyn. 103, 515.

Withbroe, G.L., Noyes, R.W.: 1977, Annu. Rev. Astron. Astrophys. 15, 363.

Yang, W.H, Sturrock, P.A., Antiochos, S.K.: 1986, Astrophys. J. 309, 383.

Yeates, A.R., Mackay, D.H., van Ballegooijen, A.A.: 2008, Solar Phys. 247, 103.

Zhou, G.P., Wang, J.X., Jin, C.L.: 2010, Solar Phys. 267, 63.

Zirin, H.: 1985, Austral. J. Phys. 38, 961. 